\title{
Asymptotics of the integrated density of states for periodic elliptic pseudo-differential operators in dimension one
}

Alexander V. Sobolev

\begin{abstract}
We consider a periodic pseudo-differential operator on the real line, which is a lower-order perturbation of an elliptic operator with a homogeneous symbol and constant coefficients. It is proved that the density of states of such an operator admits a complete asymptotic expansion at large energies. A few first terms of this expansion are found in a closed form.
\end{abstract}

\section{Introduction}

Spectral theory of periodic operators offers an abundant array of problems with regard to all aspects of spectral structure. One of such problems is the asymptotics of the (integrated) density of states $D(\lambda)$ as the spectral parameter (the energy) $\lambda$ tends to infinity. Although the study of this object presents an independent interest, it also helps to obtain other important spectral properties. For instance, the high energy asymptotics of $D(\lambda)$ provides information on the size of spectral gaps, or, studying a "generalized density of states", one can sometimes find out whether or not the number of gaps is finite, see [2], [4] and [9]. In the recent years a number of results was obtained for the density of states for the Schrödinger operator

$$
H=-\Delta+V \text {, }
$$

in $\mathrm{L}^{2}\left(\mathbb{R}^{d}\right), \quad d \geq 1$, with a real-valued function $V$, periodic with respect to a $d$-dimensional lattice $\Gamma \subset \mathbb{R}^{d}$.

2000 Mathematics Subject Classification: Primary: 35P20, 47G30, 47A55; Secondary: 81Q10.

Keywords: Periodic pseudodifferential operators, density of states. 
Below we denote by $\mathcal{O} \subset \mathbb{R}^{d}$ a standard fundamental domain of the lattice $\Gamma$. For this operator, as well as for any other elliptic self-adjoint differential operator the density of states is defined by the formula

$$
D(\lambda)=\lim _{L \rightarrow \infty} \frac{N\left(\lambda ; H_{D}^{(L)}\right)}{L^{d}} .
$$

Here $H_{D}^{(L)}$ is the restriction of $H$ to the cube $[0, L)^{d}$ with the Dirichlet boundary conditions, and $N(\lambda ; \cdot)$ is the counting function of the discrete spectrum of $H_{D}^{(L)}$. To be precise, the quantity $D(\lambda)$ is called the integrated density of states, but for the sake of brevity we call it simply the density of states. This will not cause any confusion. Calculation of the density of states $D_{0}(\lambda)$ for the unperturbed operator $H_{0}=-\Delta$ is an elementary exercise: one easily proves (see e.g. Proposition 2.1 below) that $D_{0}(\lambda)=(2 \pi)^{-d} \mathrm{w}_{d} \lambda^{d / 2}$, where we have denoted by $\mathrm{w}_{d}$ the volume of the unit ball in $\mathbb{R}^{d}$.

The first asymptotic formulas for the operator (1.1) were obtained in the case $d=1$ ([12]). It was shown that for $C^{\infty}$-smooth $2 \pi$-periodic functions $V$ the density of states admits a complete expansion in the powers of $\lambda^{-1}$ :

$$
\left\{\begin{array}{l}
D\left(\rho^{2}\right)=\frac{1}{\pi} \rho+\sum_{j=1}^{N} b_{j} \rho^{1-2 j}+O\left(\rho^{1-2(N+1)}\right), \rho \rightarrow \infty, \quad \forall N ; \\
b_{1}=-\frac{1}{4 \pi^{2}} \int_{0}^{2 \pi} V(x) d x, \quad b_{2}=-\frac{1}{16 \pi^{2}} \int_{0}^{2 \pi}|V(x)|^{2} d x .
\end{array}\right.
$$

The remaining coefficients are found via certain explicit and simple recursion relations. Note also that in addition to this formula, an asymptotics for the spectral function of $H$ was derived in [12].

The first results on the multi-dimensional Schrödinger operator were established in [13] (see also [14] for an elementary proof) for almost periodic potentials $V$. It was proved that

$$
D\left(\rho^{2}\right)=D_{0}\left(\rho^{2}\right)+O\left(\rho^{d-2}\right), \rho \rightarrow \infty .
$$

As the spectral theory of almost periodic operators is beyond the scope of this paper, for further references on the subject we refer to [5] and [14]. For periodic potentials the above formula was improved in [4] with the help of a sophisticated microlocal technique: for $\mathrm{C}^{\infty}$-smooth $V^{\prime}$ 's it was shown that $D\left(\rho^{2}\right)=D_{0}\left(\rho^{2}\right)+c_{2} \rho^{d-2}+O\left(\rho^{d-3+\epsilon}\right), \forall \epsilon>0, \quad c_{2}=-\frac{d \mathrm{w}_{d}}{2(2 \pi)^{d}|\mathcal{O}|} \int_{\mathcal{O}} V(\mathbf{x}) d \mathbf{x}$.

A further improvement was achieved even for the more general operator $(-\Delta)^{l}+V, l>1 / 2$, in [5] through the use of a suitable perturbation argument:

$$
D\left(\rho^{2 l}\right)=D_{0}\left(\rho^{2 l}\right)+c_{2, l} \rho^{d-2 l}+O\left(\rho^{d+1-4 l} \ln \rho\right), \quad c_{2, l}=c_{2} l^{-1} .
$$


The difference between the one- and multi-dimensional cases is not surprising. For $d=1$ one can carry out more or less complete perturbation analysis of the Floquet eigenvalues. On the contrary, for $d \geq 2$ the eigenvalues split in two groups behaving differently under the perturbation $V$. One group of eigenvalues behaves essentially as in the case $d=1$, and their contribution can be relatively easily evaluated by means of the perturbation theory. The analysis of the other group of eigenvalues presents a major obstacle. This group consists of "anomalous" eigenvalues which move by a quantity of order $\|V\|$ under the perturbation $V$, and therefore cannot be considered as small perturbations of the unperturbed eigenvalues (see e.g. [15], Ch. 4). In the relevant literature they are sometimes called resonant, unstable or singular eigenvalues, see [3], [6]. Various spectral properties of the operator $H$ can be established by checking that the quantity of these "anomalous" eigenvalues is negligible in some sense. For instance, this strategy was successfully applied in [15] for the justification of the Bethe-Sommerfeld conjecture, and in [5] for the proof of (1.4). On the other hand, this approach does not allow one to study further terms (if they exist) in the asymptotics of $D(\lambda)$. In spite of these difficulties at least a partial analysis of the resonant eigenvalues is possible. As was shown in [3] (see also [6], Section 4.6), they can be described by a suitable effective one-dimensional Schrödinger operator.

The aim of the present paper is to study the density of states of a periodic elliptic pseudo-differential operator in $L^{2}(\mathbb{R})$, of the form

$$
H=H_{0}+B, H_{0}=\mathrm{Op}\left(h_{0}\right), B=\mathrm{Op}(b)
$$

where $H_{0}$ is a pseudo-differential operator $(\mathrm{PDO})$ with the symbol $h_{0}(\xi)=$ $|\xi|^{m}, m>0$. The operator $B$ is a symmetric PDO of order $\alpha<m$ with a symbol $b(x, \xi)$ which is $2 \pi$-periodic in $x$. It is shown (see Theorems $2.3,2.4$ ) that the density of states admits a complete asymptotic expansion with a remainder estimate uniform in the symbol $b$ in a certain sense. A few first terms of this expansion are calculated explicitly. It is also possible to find all the subsequent coefficients as well, but the author has been able to find neither a compact formula for the coefficients, nor a simple recursion relation for them. Applying Theorem 2.3 to the Schrödinger operator one recovers the first coefficients in the decomposition (1.3) found previously in [12]. Note that the assumption that the principal symbol $h_{0}$ is $x$-independent, is not essential. A more general principal term, for instance $a(x) H_{0} a(x)$ with a positive periodic function $a(x)$, reduces to (1.5) by a simple change of variables.

Although the study of such a one-dimensional problem presents an independent interest, the main motivation comes from the link with the multidimensional case mentioned above: in a subsequent publication it is intended 
to apply the asymptotics of $D(\lambda)$ in the case $d=1$ to the study of the density of states for the multi-dimensional Schrödinger operator. For this application it would be sufficient to study the operator (1.5) of order $m=2$ and a perturbation $B$ of order $\alpha=0$, but the techniques used in the paper allow for arbitrary $m>0$ and arbitrary $\alpha<m$ without any additional effort.

The key point of the approach in the paper is to find a similarity transformation which reduces $H$ to a PDO of a convenient form. In the onedimensional case, for an elliptic PDO on the circle one can find a suitable FIO such that after the similarity transformation the operator reduces to a PDO with constant coefficients, up to a smoothing operator of order $-\infty$. This reduction is presented in a systematic way in [11] (see also survey [1] for relevant references). A further generalisation was suggested in [7], [8] and some companion papers. In the multi-dimensional case a convenient reduction is also possible in certain cases. For instance, in [16] the operator $-\Delta+V$ on the sphere $\mathbb{S}^{d}, d \geq 1$ is reduced up to terms of higher orders, to $-\Delta+\tilde{V}$ where $\tilde{V}$ is a PDO of order 0 , commuting with $-\Delta$.

Our approach can be viewed as a variant of the method described in [11]. The similarity transformation $S$ is sought in the form of a unitary operator $S=e^{i \Psi}$ in $\mathrm{L}^{2}(\mathbb{R})$ with a self-adjoint bounded periodic $\operatorname{PDO} \Psi$. The requirement that $S^{*} H S$ should be a PDO with constant coefficients leads to a series of commutator-type equations for the symbol of $\Psi$. Afterwards, the density of states for the obtained PDO with constant coefficients is found directly. As can be seen from the proof, see Sect. 4, the assumption that the principal symbol $h_{0}$ is $x$-independent, is crucial for finding $S$ in the form $e^{i \Psi}$ without the use of the FIO technique.

Let us describe briefly how the methods and results of this paper are applied to the study of the Schrödinger operator $P=-\Delta+V$ with a real-valued periodic potential $V$ in higher dimensions. For $d \geq 2$ one also constructs a similarity transformation $S$ in the form $e^{i \Psi}$. However, a complete reduction to a PDO with constant coefficients is not possible any more, since the attempt to find a suitable smooth symbol $\psi(\mathbf{x}, \boldsymbol{\xi})$ leads to a symbol singular for values of $\boldsymbol{\xi}$ on a set $\Lambda$ which is a union of hyper-planes

$$
\Lambda_{\boldsymbol{\theta}}=\left\{\boldsymbol{\xi} \in \mathbb{R}^{d}: \boldsymbol{\theta}(\boldsymbol{\xi}+\boldsymbol{\theta} / 2)=0\right\}
$$

where $\boldsymbol{\theta} \in \Gamma^{\dagger}, \Gamma^{\dagger}$ being the dual lattice. One can avoid this singularity by performing a "partial" reduction, which replaces the potential $V$ in the neighbourhood of each $\Lambda_{\boldsymbol{\theta}}$ with an appropriate zero order PDO, acting only in the variable along the direction $\boldsymbol{\theta}$. At this point the one-dimensional results apply and they eventually provide an asymptotics of the density of states for the operator $P$. Note that the set $\Lambda$ is exactly the set responsible for the existence of the resonant eigenvalues mentioned previously. 
The paper is organized as follows. Section 2 contains the precise definitions of objects studied in the paper, and the statement of the main result (see Theorems 2.3, 2.4). In Section 3 necessary information on the calculus of periodic PDO's is collected, and the perturbation $B$ is prepared for the reduction of the operator $H_{0}+B$ to a $\mathrm{PDO} A_{0}$ with constant coefficients. This reduction is implemented in Section 4. In Section 5 the integrated density of states for the operator $A_{0}$ is analyzed and the proof of Theorem 2.3 is completed.

\section{Periodic pseudo-differential operators. Main result}

\subsection{Classes of PDO's}

In order to define periodic PDO's we need first to introduce a relevant class of symbols. Although this paper is mainly concerned with the one-dimensional case, these definitions without additional cost can be given for arbitrary dimension $d \geq 1$. Let $\Gamma \in \mathbb{R}^{d}$ be a lattice. Denote by $\mathcal{O}$ its fundamental domain. For example, for $\mathcal{O}$ one can choose a parallelepiped spanned by a basis of $\Gamma$. The dual lattice and its fundamental domain are denoted by $\Gamma^{\dagger}$ and $\mathcal{O}^{\dagger}$ respectively. In particular in the case $\Gamma=(2 \pi \mathbb{Z})^{d}$ one has $\Gamma^{\dagger}=\mathbb{Z}^{d}$ and it is natural to take $\mathcal{O}=[0,2 \pi)^{d}, \mathcal{O}^{\dagger}=[0,1)^{d}$. The volume of the fundamental domain does not depend on its choice, it is called the determinant of the lattice $\Gamma$ and denoted $\mathrm{d}(\Gamma)=|\mathcal{O}|$.

For any $u \in \mathrm{L}^{2}(\mathcal{O})$ define the Fourier coefficients

$$
\hat{u}(\boldsymbol{\theta})=\frac{1}{\sqrt{\mathrm{d}(\boldsymbol{\Gamma})}} \int_{\mathcal{O}} e^{-i \boldsymbol{\theta} \mathbf{x}} u(\mathbf{x}) d \mathbf{x}, \boldsymbol{\theta} \in \Gamma^{\dagger} .
$$

Let us now define the periodic symbols and PDO's associated with them. Let $b=b(\mathbf{x}, \boldsymbol{\xi}), \mathbf{x}, \boldsymbol{\xi} \in \mathbb{R}^{d}$, be a periodic complex-valued function, that is

$$
b(\mathbf{x}+\boldsymbol{\gamma}, \boldsymbol{\xi})=b(\mathbf{x}, \boldsymbol{\xi}), \forall \boldsymbol{\gamma} \in \Gamma .
$$

Denote by $\hat{b}(\boldsymbol{\theta}, \boldsymbol{\xi}), \boldsymbol{\theta} \in \Gamma^{\dagger}$, the Fourier coefficient of the function $b(\cdot, \boldsymbol{\xi})$. Let $w: \mathbb{R}^{d} \rightarrow \mathbb{R}$ be a locally bounded function such that $w(\boldsymbol{\xi}) \geq 1, \forall \boldsymbol{\xi} \in \mathbb{R}^{d}$ and

$$
w(\boldsymbol{\xi}+\boldsymbol{\eta}) \leq C w(\boldsymbol{\xi})\langle\boldsymbol{\eta}\rangle^{\kappa}, \forall \boldsymbol{\xi}, \boldsymbol{\eta} \in \mathbb{R}^{d}
$$

for some $\kappa \geq 0$. We say that the symbol $b$ belongs to the class $\mathbf{S}_{\alpha}=$ $\mathbf{S}_{\alpha}(w), \alpha \in \mathbb{R}$, if for any $l \geq 0$ and any non-negative $s \in \mathbb{Z}$ the condition

$$
|b|_{l, s}^{(\alpha)}=\max _{p \leq l} \max _{|\mathbf{s}| \leq s} \sup _{\boldsymbol{\xi}, \boldsymbol{\theta}}\langle\boldsymbol{\theta}\rangle^{p} w(\boldsymbol{\xi})^{-\alpha+|\mathbf{s}|}\left|\mathbf{D}_{\boldsymbol{\xi}}^{\mathbf{s}} \hat{b}(\boldsymbol{\theta}, \boldsymbol{\xi})\right|<\infty,
$$


is fulfilled. Here we have used the standard notation $\langle\mathbf{t}\rangle=\sqrt{1+|\mathbf{t}|^{2}}, \forall \mathbf{t} \in$ $\mathbb{R}^{d}$. Also, for any $\mathbf{s} \in \mathbb{Z}^{d}$ we denote $|\mathbf{s}|=s_{1}+s_{2}+\cdots+s_{d}$. We mainly use classes $\mathbf{S}_{\alpha}$ with the weight $w(\boldsymbol{\xi})=\langle\boldsymbol{\xi}\rangle$, which satisfies (2.1) for $\kappa=1$. Note that $\mathbf{S}_{\alpha}$ is an increasing function of $\alpha$, i.e. $\mathbf{S}_{\alpha} \subset \mathbf{S}_{\beta}$ for $\alpha<\beta$. For later reference write the following convenient bounds that follow from definition (2.2) and property (2.1):

$$
\begin{aligned}
\left|\mathbf{D}_{\xi}^{\mathbf{s}} \hat{b}(\boldsymbol{\theta}, \boldsymbol{\xi})\right| & \leq|b|_{l, s}^{(\alpha)}\langle\boldsymbol{\theta}\rangle^{-l} w(\boldsymbol{\xi})^{\alpha-s} \\
\left|\mathbf{D}_{\boldsymbol{\xi}}^{\mathrm{s}} \hat{b}(\boldsymbol{\theta}, \boldsymbol{\xi}+\boldsymbol{\eta})-\mathbf{D}_{\boldsymbol{\xi}}^{\mathbf{s}} \hat{b}(\boldsymbol{\theta}, \boldsymbol{\xi})\right| \leq & \leq C|b|_{l, s+1}^{(\alpha)}\langle\boldsymbol{\theta}\rangle^{-l} w(\boldsymbol{\xi})^{\alpha-s-1}\langle\boldsymbol{\eta}\rangle^{\kappa|\alpha-s-1|}|\boldsymbol{\eta}|, s=|\mathbf{s}|,
\end{aligned}
$$

with a constant $C$ depending only on $\alpha, s$. For any symbol $b \in \mathbf{S}_{\alpha}$ we define the $\operatorname{PDO} \mathrm{Op}(b)$ in the usual way:

$$
\mathrm{Op}(b) u(\mathbf{x})=\frac{1}{(2 \pi)^{d}} \iint b(\mathbf{x}, \boldsymbol{\xi}) e^{i \boldsymbol{\xi}(\mathbf{x}-\mathbf{y})} u(\mathbf{y}) d \mathbf{y} d \boldsymbol{\xi},
$$

the integrals being over $\mathbb{R}^{d}$. One can easily see that under the condition $|b|_{l, 0}^{(\alpha)}<\infty, l>d$, the r.h.s. is finite for any $u$ from the Schwarz class $\mathrm{S}\left(\mathbb{R}^{d}\right)$. Moreover, later we show that for $\alpha=0$ this condition guarantees the boundedness of $\mathrm{Op}(b)$ in $\mathrm{L}^{2}\left(\mathbb{R}^{d}\right)$. From now on $\mathrm{S}\left(\mathbb{R}^{d}\right)$ is taken as a natural domain for all PDO's at hand. Observe that the operator $\mathrm{Op}(b)$ is symmetric if its symbol satisfies the condition

$$
\hat{b}(\boldsymbol{\theta}, \boldsymbol{\xi})=\overline{\hat{b}(-\boldsymbol{\theta}, \boldsymbol{\xi}+\boldsymbol{\theta})} .
$$

We shall call such symbols symmetric.

For methodological purposes, apart from $\mathbf{S}_{\alpha}$ we also need a class of symbols, depending on a scalar parameter $\sigma \geq \sigma_{0}>0$ in a controllable way. To keep things as simple as possible, we allow only the dependence on $\sigma$ which will be needed for our argument later. Assume that $b=b(\mathbf{x}, \boldsymbol{\xi} ; \sigma)$ is a $\mathbf{C}^{\infty}$-function of $\sigma \geq \sigma_{0}$, such that $\partial_{\sigma}^{n} b(\cdot, \cdot ; \sigma) \in \mathbf{S}_{\alpha}$ for each $\sigma \geq \sigma_{0}$ and

$$
\mathbf{|} b \mathbf{|}_{l, s, r}^{(\alpha, \delta)}:=\max _{n \leq r} \sup _{\sigma \geq \sigma_{0}} \sigma^{n-\delta} \mathbf{|} \partial_{\sigma}^{n} b(\cdot, \cdot ; \sigma) \mathbf{|}_{l, s}^{(\alpha)}<\infty,
$$

for some $\beta \in \mathbb{R}$ and all non-negative integers $l, s, r$. The class of such symbols is denoted by $\mathbf{T}_{\alpha, \delta}=\mathbf{T}_{\alpha, \delta}\left(w ; \sigma_{0}\right)$. Clearly, $\mathbf{T}_{\alpha, \delta} \subset \mathbf{T}_{\beta, \zeta}$ if $\alpha \leq \beta, \delta \leq \zeta$. It is natural to identify the original class $\mathbf{S}_{\alpha}$ with a subset of $\mathbf{T}_{\alpha, 0}$ which consist of symbols constant in $\sigma$. When it is not confusing, sometimes we omit the dependence of symbols $b \in \mathbf{T}_{\alpha, \delta}$ on the parameter $\sigma$. Note that similarly to (2.3), one can deduce from (2.6) that

$$
\left|\partial_{\sigma}^{n} \mathbf{D}_{\xi}^{\mathbf{s}} \hat{b}(\boldsymbol{\theta}, \boldsymbol{\xi} ; \sigma)\right| \leq|b|_{l, s, n}^{(\alpha, \delta)}\langle\boldsymbol{\theta}\rangle^{-l} w(\boldsymbol{\xi})^{\alpha-s} \sigma^{\delta-n}
$$


We are interested in spectral properties of the operator $H=H_{0}+B, B=$ $\mathrm{Op}(b)$ in $\mathcal{H}=\mathrm{L}^{2}\left(\mathbb{R}^{d}\right)$. Here $b \in \mathbf{S}_{\alpha}(w), w(\boldsymbol{\xi})=\langle\boldsymbol{\xi}\rangle$, is a symmetric symbol, $H_{0}=\mathrm{Op}\left(h_{0}\right)$ with the symbol $h_{0}(\boldsymbol{\xi})=|\mathbf{F} \boldsymbol{\xi}|^{m}$, where $m>\alpha$ and $\mathbf{F}$ is a non-degenerate matrix having real valued entries. Clearly, $H_{0}$ is self-adjoint on the domain $D\left(H_{0}\right)=\mathrm{H}^{m}\left(\mathbb{R}^{d}\right)$. As we shall see later, any PDO with a symbol $b \in \mathbf{S}_{\alpha}, \alpha<m$ is $H_{0}$-bounded with the relative bound zero. More precisely, under the condition $|b|_{l, 0}^{(\alpha)}<\infty, l>d$ we have

$$
\|B u\| \leq \epsilon\left\|H_{0} u\right\|+C_{\epsilon}\|u\|, \forall \epsilon>0,
$$

with $C_{\epsilon}=C_{\epsilon}(b)$. Hence the operator $H=H_{0}+B$ is also self-adjoint on $D(H)=D\left(H_{0}\right)$. Due to the $\Gamma$-periodicity of the symbol $b$, the operator $H$ commutes with the shifts along the lattice vectors, i.e.

$$
H T_{\gamma}=T_{\gamma} H, \gamma \in \Gamma \text {. }
$$

with $\left(T_{\boldsymbol{\gamma}} u\right)(\mathbf{x})=u(\mathbf{x}+\boldsymbol{\gamma})$. This allows us to use the Floquet decomposition.

\subsection{Floquet decomposition}

We identify the space $\mathcal{H}$ with the direct integral

$$
\mathfrak{G}=\int_{\mathcal{O}^{\dagger}} \mathfrak{H} d \mathbf{k}, \mathfrak{H}=\mathrm{L}^{2}(\mathcal{O})
$$

This identification is implemented by the Gelfand transform

$$
(U u)(\mathbf{x}, \mathbf{k})=\frac{1}{\sqrt{d\left(\Gamma^{\dagger}\right)}} e^{-i\langle\mathbf{k}, \mathbf{x}\rangle} \sum_{\gamma \in \Gamma} e^{-i\langle\mathbf{k}, \boldsymbol{\gamma}\rangle} u(\mathbf{x}+\boldsymbol{\gamma}), \mathbf{k} \in \mathcal{O}^{\dagger},
$$

which is initially defined on $u \in \mathrm{S}\left(\mathbb{R}^{d}\right)$ and extends by continuity to a unitary mapping from $\mathcal{H}$ onto $\mathfrak{G}$. The unitary operator $U$ reduces $T_{\boldsymbol{\gamma}}$ to the diagonal form:

$$
\left(U T_{\gamma} U^{-1} f\right)(\cdot, \mathbf{k})=e^{i\langle\mathbf{k}, \boldsymbol{\gamma}\rangle} f(\cdot, \mathbf{k}), \forall \boldsymbol{\gamma} \in \Gamma .
$$

Let us consider a self-adjoint operator $A$ in $\mathcal{H}$ which commutes with $T_{\gamma}$ for all $\gamma \in \Gamma$. Then $A$ is partially diagonalised by $U$ (see [10]), that is, there exists a measurable family of self-adjoint operators $A(\mathbf{k})$ acting in $\mathfrak{H}$, such that

$$
U A U^{*}=\int_{\mathcal{O}^{\dagger}} A(\mathbf{k}) d \mathbf{k} .
$$

It is easy to show that any periodic operator $T$, which is $A$-bounded with relative bound $\epsilon<1$, can be also decomposed into a measurable set of fibers $T(\mathbf{k})$ in the sense that

$$
(U T f)(\cdot, \mathbf{k})=T(\mathbf{k})(U f)(\cdot, \mathbf{k}), \text { a.e. } \mathbf{k} \in \mathcal{O}^{\dagger},
$$


for all $f \in D(A)$. Moreover, the fibers $T(\mathbf{k})$ are $A(\mathbf{k})$-bounded with the bound $\epsilon$, and if $T$ is symmetric, then the operator $A(\mathbf{k})+T(\mathbf{k})$ is self-adjoint on $D(A(\mathbf{k}))$.

If $A=\mathrm{Op}(a)$ with a real-valued symbol $a \in \mathrm{L}_{\mathrm{loc}}^{\infty}\left(\mathbb{R}^{d}\right)$ depending only on $\boldsymbol{\xi}$, then $A(\mathbf{k})$ is a self-adjoint PDO on the torus $\mathbb{T}^{d}=\mathbb{R}^{d} / \Gamma$ defined as follows:

$$
A(\mathbf{k}) u(\mathbf{x})=\frac{1}{\sqrt{\mathrm{d}(\Gamma)}} \sum_{\boldsymbol{\mu} \in \Gamma^{\dagger}} e^{i\langle\boldsymbol{\mu}, \mathbf{x}\rangle} a(\boldsymbol{\mu}+\mathbf{k}) \hat{u}(\boldsymbol{\mu}) .
$$

If $a(\boldsymbol{\xi}) \rightarrow \infty$ as $|\boldsymbol{\xi}| \rightarrow \infty$, then the spectrum of each $A(\mathbf{k})$ is purely discrete with eigenvalues given by $\lambda^{(\mathbf{b})}(\mathbf{k})=a(\mathbf{b}+\mathbf{k}), \mathbf{b} \in \Gamma^{\dagger}$. Consequently, the number of eigenvalues below each $\lambda \in \mathbb{R}$ is essentially bounded from above uniformly in $\mathbf{k} \in \mathcal{O}^{\dagger}$. If $T$ is a periodic symmetric operator which is $A$ bounded with a bound $\epsilon<1$, then the spectrum of $A(\mathbf{k})+T(\mathbf{k})$ is also purely discrete and the counting function is also bounded uniformly in $\mathbf{k}$.

The above applies to the operators $A=H_{0}=\mathrm{Op}\left(h_{0}\right)$ with $h_{0}(\boldsymbol{\xi})=|\mathbf{F} \boldsymbol{\xi}|^{m}$ and $T=B=\mathrm{Op}(b)$ with a symmetric symbol $b \in \mathbf{S}_{\alpha}(w), w=\langle\boldsymbol{\xi}\rangle, \alpha<m$. In, particular the spectrum of $H(\mathbf{k})=H_{0}(\mathbf{k})+B(\mathbf{k})$ is purely discrete, with eigenvalues $\lambda_{j}(\mathbf{k})$ enumerated in increasing order counting multiplicity. Moreover, the counting function

$$
N(\lambda, \mathbf{k})=\#\left\{j: \lambda_{j}(\mathbf{k}) \leq \lambda\right\}, \lambda \in \mathbb{R}
$$

satisfies $N(\lambda, \cdot) \in \mathrm{L}^{\infty}\left(\mathcal{O}^{\dagger}\right)$. Let us introduce the notation for the Fourier coefficients of this function:

$$
\hat{N}(\lambda, \mathbf{b})=\frac{1}{(2 \pi)^{d}} \int_{\mathcal{O}^{\dagger}} e^{-i\langle\mathbf{b}, \mathbf{k}\rangle} N(\lambda, \mathbf{k}) d \mathbf{k}, \mathbf{b} \in \Gamma .
$$

The zeroth coefficient is called the (integrated) density of states for the operator $H$ :

$$
D(\lambda)=\hat{N}(\lambda, \mathbf{0})=\frac{1}{(2 \pi)^{d}} \int_{\mathcal{O}^{\dagger}} N(\lambda, \mathbf{k}) d \mathbf{k} .
$$

For periodic differential elliptic operators this is equivalent to the standard definition (1.2) of the density of states, see [14]. For general PDO's the formula (1.2) is not applicable, and thus (2.11) is used as a definition. Sometimes we need to reflect the dependence of the counting function and density of states on the operator. In this case we use the notation $N(\lambda, \mathbf{k} ; H), D(\lambda ; H), \hat{N}(\lambda, \mathbf{b} ; H)$.

In fact, if necessary, one can obtain more information on the operators $H_{0}(\mathbf{k})$ and $B(\mathbf{k})$. First of all, using the fact that the symbol of $H_{0}(\mathbf{k})$ is $|\mathbf{F}(\boldsymbol{\mu}+\mathbf{k})|^{m}$ one concludes that $D\left(H_{0}(\mathbf{k})\right)=\mathbf{H}^{m}\left(\mathbb{T}^{d}\right)$. Secondly, applying 
the Gelfand transform $(2.9)$ to $\mathrm{Op}(b)$, one finds that, similarly to $H_{0}(\mathbf{k})$, the operator $B(\mathbf{k})$ is a $\mathrm{PDO}$ on the torus of the form

$$
B(\mathbf{k}) u(\mathbf{x})=\frac{1}{\sqrt{\mathrm{d}(\Gamma)}} \sum_{\boldsymbol{\mu} \in \Gamma^{\dagger}} e^{i\langle\boldsymbol{\mu}, \mathbf{x}\rangle} b(\mathbf{x}, \boldsymbol{\mu}+\mathbf{k}) \hat{u}(\boldsymbol{\mu}) .
$$

Moreover, if the symbol $b(\mathbf{x}, \boldsymbol{\xi})$ is smooth in $\boldsymbol{\xi}$, then the family $B(\mathbf{k})$ is smooth in $\mathbf{k}$. Note however that for our purposes we need neither this explicit formula, nor the smoothness property.

We shall need one more notational convention. For any measurable set $\mathcal{C} \subset \mathbb{R}^{d}$ we denote by $\chi(\cdot, \mathcal{C})$ the characteristic function of $\mathcal{C}$. Then the operator

$$
\mathcal{P}=\mathcal{P}(\mathcal{C})=\mathrm{Op}(\chi(\boldsymbol{\xi}, \mathcal{C}))
$$

is a projection in $\mathcal{H}$. Moreover, $\mathcal{P} D(H) \subset D(H)$. Since the operator $\mathcal{P}$ commutes with $T_{\boldsymbol{\gamma}}$, it is also decomposable, and similarly to $H(\mathbf{k})$, the operator

$$
(\mathcal{P} H \mathcal{P})(\mathbf{k})=\mathcal{P}(\mathbf{k}) H(\mathbf{k}) \mathcal{P}(\mathbf{k}),
$$

restricted to the subspace $\mathcal{P}(\mathbf{k}) \mathfrak{H}$ has discrete spectrum. For the counting function of this spectrum we use the notation $N(\lambda, \mathbf{k} ; \mathcal{P} H \mathcal{P})$.

The quantity $\hat{N}(\lambda, \mathbf{b} ; \mathrm{Op}(a))$ has a transparent geometrical meaning if the symbol $a$ does not depend on $\mathbf{x}$. We state the appropriate formulas in the form of a proposition for later reference.

Proposition 2.1 Let $A=\mathrm{Op}(a)$ with a real-valued symbol $a \in \mathrm{L}_{\mathrm{loc}}^{\infty}\left(\mathbb{R}^{d}\right)$ be such that $a(\boldsymbol{\xi}) \rightarrow \infty$ as $|\boldsymbol{\xi}| \rightarrow \infty$. Then for any $\lambda \in \mathbb{R}$

$$
\hat{N}(\lambda, \mathbf{b} ; A)=\frac{1}{(2 \pi)^{d}} \int_{\xi: a(\boldsymbol{\xi}) \leq \lambda} e^{-i \mathbf{b} \boldsymbol{\xi}} d \boldsymbol{\xi} .
$$

In particular, $(2 \pi)^{d} D(\lambda ; A)$ equals the volume of the body $\{\boldsymbol{\xi}: a(\boldsymbol{\xi}) \leq \lambda\}$.

Proof. Let us prove the formula for $D(\lambda)$. Denote

$$
\Theta(t)=\left\{\begin{array}{l}
0, t<0 \\
1, t \geq 0
\end{array}\right.
$$

Observe that the eigenvalues of $A(\mathbf{k})$ equal $a(\boldsymbol{\mu}+\mathbf{k}), \boldsymbol{\mu} \in \Gamma^{\dagger}$, so that

$$
\begin{aligned}
(2 \pi)^{d} D(\lambda) & =\sum_{\boldsymbol{\mu} \in \Gamma^{\dagger}} \int_{\mathcal{O}^{\dagger}} \Theta(\lambda-a(\boldsymbol{\mu}+\mathbf{k})) d \mathbf{k} \\
& =\int_{a(\boldsymbol{\xi}) \leq \lambda} d \boldsymbol{\xi}=\operatorname{vol}\left(\boldsymbol{\xi} \in \mathbb{R}^{d}: a(\boldsymbol{\xi}) \leq \lambda\right),
\end{aligned}
$$

as required.

Note the invariance of the density of states under certain unitary transformations: 
Lemma 2.2 Let $W: \mathcal{H} \rightarrow \mathcal{H}$ be a $\Gamma$-periodic unitary operator, i.e.

$$
W T_{\gamma}=T_{\gamma} W, \forall \gamma \in \Gamma .
$$

Then the operator $W$ is also decomposable in the direct integral, with fibres $W(\mathbf{k})$ that are unitary operators in $\mathfrak{H}$. In particular

$$
N\left(\lambda, \mathbf{k} ; W^{*} H W\right)=N(\lambda, \mathbf{k} ; H)
$$

and

$$
D(\lambda ; H)=D\left(\lambda ; W^{*} H W\right), \quad \hat{N}(\lambda, \mathbf{b} ; H)=\hat{N}\left(\lambda, \mathbf{b} ; W^{*} H W\right), \quad \forall \mathbf{b} \in \Gamma .
$$

\subsection{Main result}

The present paper deals with the asymptotics of the density of states $D(\lambda)$ as $\lambda \rightarrow \infty$ for the case $d=1$. The perturbation $\mathrm{Op}(b)$ is supposed to belong to the class $\mathbf{S}_{\alpha}(w)$ with $w(\xi)=\langle\xi\rangle$ and $\alpha<m$. When $d=1$ we always assume for convenience that $\Gamma=2 \pi \mathbb{Z}$. We prove the existence of an asymptotic expansion for $\hat{N}(\lambda, 2 \pi n), n \in \mathbb{Z}$, as $\lambda \rightarrow \infty$ for the one-dimensional case, and calculate explicitly a few first terms of this asymptotics. However, a number of auxiliary results will be established for general $d \geq 1$. To emphasise the difference between $d=1$ and $d>1$ we use the notation $x, \xi$ and $k$ in the case $d=1$ instead of $\mathbf{x}, \boldsymbol{\xi}$ and $\mathbf{k}$ for $d>1$. Denote

$$
\mathcal{B}_{1}(\xi)=\int_{0}^{2 \pi} b(x, \xi) d x, \quad \mathcal{B}_{2}(\xi)=\int_{0}^{2 \pi}|b(x, \xi)|^{2} d x
$$

Before stating the result we need a few more definitions. Similarly to the class $\mathbf{T}_{\alpha, \delta}\left(w ; \sigma_{0}\right)$ of PDO's depending on the parameter $\sigma$, we also introduce the class

$$
\mathcal{T}_{j, \delta}^{\nu}=\mathcal{T}_{j, \delta}^{\nu}\left(\xi_{0}, \sigma_{0}\right) \subset \mathrm{C}^{\infty}\left(\mathbb{R} \backslash\left(-\xi_{0}, \xi_{0}\right) \times\left[\sigma_{0}, \infty\right)\right), \xi_{0} \geq 0, \sigma_{0}>0,
$$

of functions $\mu=\mu(\xi ; \sigma)$ such that

$$
\partial_{\sigma}^{n} \partial_{\xi}^{s} \mu(\xi ; \sigma) \leq C_{s, n}\langle\xi\rangle^{(\delta-\varkappa) j+\nu-s} \sigma^{-n}, \varkappa=m-\alpha>0,
$$

for all non-negative integer $s, n$. A function $\mu$ is said to belong to $\mathcal{T}_{j, \delta}^{\nu}$ uniformly in the symbol $b \in \mathbf{T}_{\alpha, \beta}$ (or $b \in \mathbf{S}_{\alpha}$ ) if the constants $C_{s, n}$ in (2.12) depend only on the constants in the estimates $|b|_{l, s, r}^{(\alpha, \delta)} \leq C_{l, s, r}$. We say that the function $a(\xi ; \sigma)$ is represented by an asymptotic series $\sum_{j=1}^{\infty} \mu_{j}(\xi ; \sigma)$ with $\mu_{j} \in \mathcal{T}_{j, \delta}^{\nu}$ if

$$
u_{M+1}^{(\nu)}(\xi ; \sigma ; a):=a(\xi ; \sigma)-\sum_{j=1}^{M} \mu_{j}(\xi ; \sigma)=O\left(\langle\xi\rangle^{(M+1)(\delta-\varkappa)+\nu}\right),
$$


for all $M \in \mathbb{N}$. We write this down as follows: $a(\xi ; \sigma)=\sum_{j=1}^{\infty} \mu_{j}(\xi ; \sigma)$. We say that that a function $\omega$ belongs to a class $\mathcal{T}_{k, \delta}^{\epsilon}$ uniformly in the series $a$, if the estimates of the form (2.12) for $\omega$ are satisfied with constants $C_{s, n}$ depending only on the constants in corresponding estimates for the functions $\mu_{j}$ and for the remainder $u_{M+1}^{(\nu)}(\xi ; \sigma ; a)$.

Suppose that another function $g(\xi ; \sigma)$ is represented by an asymptotic series

$$
g(\xi ; \sigma)=\sum_{j=1}^{\infty} \omega_{j}(\xi ; \sigma)
$$

with $\omega_{j} \in \mathcal{T}_{j, \delta}^{\epsilon}$. Then we say that this series is uniform with respect to the series $a(\xi ; \sigma)$, if the functions $\omega_{j}$ belong to $\mathcal{T}_{j, \delta}^{\epsilon}$ uniformly in $a$, and the remainder $u_{M+1}^{(\epsilon)}(\xi ; \sigma ; g)$ satisfies (2.13) uniformly in $a$.

By $\mathcal{S}_{j, \delta}^{\nu} \subset \mathcal{T}_{j, \delta}^{\nu}\left(0, \sigma_{0}\right)$ we denote the set of functions $\mu \in \mathcal{T}_{j, \delta}^{\nu}$ depending only on the variable $\xi \in \mathbb{R}$.

The main result is contained in the following Theorem:

Theorem 2.3 Let $m>0$. Let $b \in \mathbf{S}_{\alpha}(w), w(\xi)=\langle\xi\rangle$, be a symmetric $2 \pi$-periodic symbol with $\alpha<m$. Then there exists a sequence of functions $\nu_{j}, j=1,2, \ldots$, that belong to $\mathcal{S}_{j, 0}^{1}$ uniformly in $b$, such that the sum

$$
\xi_{M}(\rho)=\rho+\sum_{j=1}^{M} \nu_{j}(\rho)
$$

describes the asymptotics of the density of states and of the other Fourier coefficients of the counting function $N\left(\rho^{m}, \mathbf{k}\right)$ for all $\rho \geq 0$, any $M \in \mathbb{N}$ and $n \in \mathbb{Z} \backslash\{0\}$ by the formulas

$$
\begin{gathered}
D\left(\rho^{m}\right)=\frac{1}{2 \pi}\left[\xi_{M}(\rho)-\xi_{M}(-\rho)\right]+O\left(\langle\rho\rangle^{-(M+1) \varkappa+1}\right), \varkappa=m-\alpha \\
\hat{N}\left(\rho^{m} ; 2 \pi n\right)=-\frac{1}{4 \pi^{2} i n}\left(\exp \left(-2 \pi i n \xi_{M}(\rho)\right)-\exp \left(-2 \pi i n \xi_{M}(-\rho)\right)\right) \\
+O\left(\langle\rho\rangle^{-(M+1) \varkappa+1}\right) .
\end{gathered}
$$

The first two functions $\nu_{j}$ are given by the formulas

$$
\begin{aligned}
& \nu_{1}(\rho)=-\frac{1}{2 \pi m} \rho|\rho|^{-m} \mathcal{B}_{1}(\rho), \\
& \nu_{2}(\rho)=-\frac{1}{4 \pi m^{2}} \rho^{-2 m+1}\left[(m-1) \mathcal{B}_{2}(\rho)-\rho \mathcal{B}_{2}^{\prime}(\rho)\right]+O\left(\langle\rho\rangle^{-2 \varkappa-1}\right),
\end{aligned}
$$

$|\rho| \geq 2$. The asymptotics (2.14) and (2.15) and the formulas for $\nu_{1}$ and $\nu_{2}$ are uniform in $b$ in the sense that the remainders depend only on the constants in the bounds $|b|_{l, s}^{(\alpha)} \leq C_{l, s}$. Besides, the remainder in (2.15) is uniform in $n \in \mathbb{Z} \backslash\{0\}$. 
The above Theorem follows from the next Theorem, in which the asymptotics of $D\left(\rho^{m}\right)$ is studied for a symbol $b$ depending on $\rho$ :

Theorem 2.4 Let $m>0$. Let $b \in \mathbf{T}_{\alpha, \delta}(w), w(\xi)=\langle\xi\rangle$, be a symmetric $2 \pi$ periodic symbol $b(x, \xi)=b(x, \xi ; \rho)$ with $\alpha_{1}+\delta<m$ where $\alpha_{1}=\max (0, \alpha)$. Then there exists a sequence of functions $\nu_{j}, j=1,2, \ldots$, that belong to $\mathcal{S}_{j, \delta}^{1}$ uniformly in $b$, such that the asymptotics of the density of states and of the other Fourier coefficients of the counting function $N\left(\rho^{m}, \mathbf{k}\right)$ for all $\rho \geq 0$, any $M \in \mathbb{N}$ and $n \in \mathbb{Z} \backslash\{0\}$ is described by the formulas (2.14) and (2.15), in which the remainder estimates are replaced by $O\left(\langle\rho\rangle^{M(\delta-\varkappa)+1}\right)$. The first two functions $\nu_{j}$ are given by the formulas (2.16) and (2.17), in the latter formula the remainder being replaced by $O\left(\langle\rho\rangle^{2(\delta-\varkappa)-1}\right)$. The asymptotics (2.14) and (2.15) and the formulas for $\nu_{1}$ and $\nu_{2}$ are uniform in $b$ in the sense that the remainders depend only on the constants in the bounds $|b|_{l, s, r}^{(\alpha, \delta)} \leq C_{l, s, r}$. Besides, the remainder in (2.15) is uniform in $n \in \mathbb{Z} \backslash\{0\}$.

Remark 2.5 Let us observe that the formulas (2.14) and (2.15) have the asymptotic meaning only for large $\rho$. For bounded $\rho$ these formulas simply say that the density of states is bounded uniformly in $b$. The latter fact can be inferred from Proposition 2.1 without any sophisticated analysis. Indeed, getting slightly ahead, and using (3.3) with $\sigma=\rho$, we conclude that

$$
|(\mathrm{Op}(b) u, u)| \leq C\left(\left(H_{0}+\rho^{m}\right) u, u\right) .
$$

Here we have used that $\alpha+\delta<m$. Consequently,

$$
\left|\hat{N}\left(\rho^{m}, 2 \pi n ; H_{0}+B\right)\right| \leq D\left(\rho^{m} ; H_{0}+B\right) \leq D\left(c \rho^{m} ; \tilde{C} H_{0}\right) .
$$

By Proposition 2.1 the r.h.s. is bounded from above uniformly in $b$ for $\rho \leq C$.

Remark that for the Schrödinger operator $H=-d^{2} / d x^{2}+V$ with a periodic potential $V \in \mathrm{C}^{\infty}$ the asymptotics obtained in Theorem 2.3 give

$$
D\left(\rho^{2}\right)=\frac{1}{\pi} \rho-\frac{1}{4 \pi^{2} \rho} \int_{0}^{2 \pi} V(x) d x-\frac{1}{16 \pi^{2} \rho^{3}} \int_{0}^{2 \pi}|V(x)|^{2} d x+O\left(\rho^{-5}\right) .
$$

This formula agrees with the first three terms of the complete asymptotic expansion established in the paper [12] for this case. Note also that the term with $O\left(\rho^{-4}\right)$ is absent, as in [12]. For the Fourier coefficients in the case $b(x, \xi)=V(x)$ Theorem 2.3 gives:

$$
\hat{N}\left(\rho^{2} ; 2 \pi n\right)=\frac{1}{2 \pi^{2} n} \sin \left[n\left(2 \pi \rho-\frac{1}{2 \rho} \int_{0}^{2 \pi} V(x) d x-\frac{1}{8 \rho^{3}} \int_{0}^{2 \pi}|V(x)|^{2} d x\right)\right]+O\left(\rho^{-5}\right) \text {. }
$$




\section{Periodic pseudo-differential operators}

\subsection{Elementary results for periodic PDO's}

As was indicated in the previous section, the calculus of PDO's is the main technical tool. In this section we collect the required properties of PDO's for arbitrary $d \geq 1$. Most of them, morally speaking, are quite standard, but nevertheless we provide full proofs. Recall that $S\left(\mathbb{R}^{d}\right)$ is taken as a natural domain of $\mathrm{Op}(b)$. Unless otherwise stated, all symbols are supposed to belong to the class $\mathbf{S}_{\alpha}=\mathbf{S}_{\alpha}(w)$ or $\mathbf{T}_{\alpha, \beta}=\mathbf{T}_{\alpha, \beta}\left(w ; \sigma_{0}\right)$ with an arbitrary function $w$ satisfying (2.1), the function $w$ and the parameter $\sigma$ being usually omitted from the notation. Recall that the class $\mathbf{S}_{\alpha}$ is naturally viewed as a subset of $\mathbf{T}_{\alpha, 0}$ of symbols constant in $\sigma$.

Let us first prove a test of boundedness, which will be systematically used:

Lemma 3.1 Assume that $|b|_{l, 0,0}^{(0, \beta)}<\infty$ with some $\beta \in \mathbb{R}$ and $l>d$. Then $B=\mathrm{Op}(b)$ is bounded in $\mathcal{H}$ and $\|B\| \leq C \sigma^{\beta} \mid b \mathbf{|}_{l, 0,0}^{(0)}$ with a constant $C=C_{l}$ independent of $b$.

Proof. The Lemma is obvious for the symbols with one non-trivial Fourier coefficient. The general case follows by summation using the bound (2.7) with $l>d$.

Since $\mathrm{Op}(b) u \in \mathbf{S}\left(\mathbb{R}^{d}\right)$ for any $b \in \mathbf{T}_{\alpha, \delta}$ and $u \in \mathbf{S}\left(\mathbb{R}^{d}\right)$, the product $\operatorname{Op}(b) \operatorname{Op}(g), b \in \mathbf{T}_{\alpha, \delta}, g \in \mathbf{T}_{\beta, \gamma}$, is well defined on $\mathbf{S}\left(\mathbb{R}^{d}\right)$. A straightforward calculation leads to the following formula for the symbol $b \circ g$ of the product $\mathrm{Op}(b) \mathrm{Op}(g)$ :

$$
(b \circ g)(\mathbf{x}, \boldsymbol{\xi})=\frac{1}{\mathrm{~d}(\Gamma)} \sum_{\boldsymbol{\theta}, \boldsymbol{\phi}} \hat{b}(\boldsymbol{\theta}, \boldsymbol{\xi}+\boldsymbol{\phi}) \hat{g}(\boldsymbol{\phi}, \boldsymbol{\xi}) e^{i(\boldsymbol{\theta}+\boldsymbol{\phi}) \mathbf{x}},
$$

and hence

$$
\widehat{(b \circ g)}(\boldsymbol{\chi}, \boldsymbol{\xi})=\frac{1}{\sqrt{\mathrm{d}(\Gamma)}} \sum_{\boldsymbol{\theta}+\boldsymbol{\phi}=\boldsymbol{\chi}} \hat{b}(\boldsymbol{\theta}, \boldsymbol{\xi}+\boldsymbol{\phi}) \hat{g}(\boldsymbol{\phi}, \boldsymbol{\xi}), \quad \boldsymbol{\chi} \in \Gamma^{\dagger}, \boldsymbol{\xi} \in \mathbb{R}^{d} .
$$

Lemma 3.2 Let $b \in \mathbf{T}_{\alpha, \delta}, g \in \mathbf{T}_{\beta, \gamma}$. Then $b \circ g \in \mathbf{T}_{\alpha+\beta, \delta+\gamma}$ and

$$
\mathbf{|} b \circ g \mathbf{|}_{p, s, r}^{(\alpha+\beta, \delta+\gamma)} \leq C_{l, s, r}\left|b \mathbf{|}_{l, s, r}^{(\alpha, \delta)}\right| g \mathbf{I}_{l, s, r}^{(\beta, \gamma)}
$$

for some $l=l(p, s)$. 
Proof. We omit the dependence on $\sigma$ from the notation. Let $s=|\mathbf{s}|$. By (3.1) and (2.7)

$$
\begin{aligned}
&\left|\partial_{\sigma}^{n} \mathbf{D}_{\boldsymbol{\xi}}^{\mathbf{s}}(b \circ g)(\boldsymbol{\chi}, \boldsymbol{\xi})\right| \leq \\
& \leq C_{s} \sum_{\boldsymbol{\theta}+\boldsymbol{\phi}=\boldsymbol{\chi}} \sum_{n_{1}+n_{2}=n} \sum_{\left|\mathbf{s}_{1}\right|+\left|\mathbf{s}_{2}\right|=s}\left|\partial_{\sigma}^{n_{1}} \mathbf{D}_{\boldsymbol{\xi}}^{\mathbf{s}_{1}} \hat{b}(\boldsymbol{\theta}, \boldsymbol{\xi}+\boldsymbol{\phi})\right|\left|\partial_{\sigma}^{n_{2}} \mathbf{D}_{\boldsymbol{\xi}}^{\mathbf{s}_{2}} \hat{g}(\boldsymbol{\phi}, \boldsymbol{\xi})\right| \\
& \leq C_{s, l, n}|b|_{l, s, n}^{(\alpha, \delta)} \mid g \mathbf{|}_{l, s, n}^{(\beta, \gamma)} \sigma^{\delta+\gamma-n} \times \\
& \quad \times \sum_{\boldsymbol{\theta}+\boldsymbol{\phi}=\boldsymbol{\chi}} \sum_{s_{1}+s_{2}=s}\langle\boldsymbol{\theta}\rangle^{-l}\langle\boldsymbol{\phi}\rangle^{-l} w(\boldsymbol{\xi}+\boldsymbol{\phi})^{\alpha-s_{1}} w(\boldsymbol{\xi})^{\beta-s_{2}} .
\end{aligned}
$$

By (2.1)

$$
w(\boldsymbol{\xi}+\phi)^{\alpha-s_{1}} \leq C\langle\boldsymbol{\phi}\rangle^{\kappa(|\alpha|+s)} w(\boldsymbol{\xi})^{\alpha-s_{1}},
$$

and hence we have

$$
\begin{aligned}
\mathbf{|} b \circ g \mathbf{|}_{p, s, r}^{(\alpha+\beta, \delta+\gamma)} & \leq C_{s, l, r}\left|b \mathbf{|}_{l, s, r}^{(\alpha, \delta)}\right| g \mathbf{|}_{l, s, r}^{(\beta, \gamma)} \sum_{\boldsymbol{\theta}, \boldsymbol{\phi}}\left(\langle\boldsymbol{\theta}\rangle^{p}+\langle\boldsymbol{\phi}\rangle^{p}\right)\langle\boldsymbol{\theta}\rangle^{-l}\langle\boldsymbol{\phi}\rangle^{-l+\kappa(|\alpha|+s)} \\
& \leq C_{l, s, r}^{\prime} \mathbf{|}|b|_{l, s, r}^{(\alpha, \delta)} \mid g \mathbf{|}_{l, s, r}^{(\beta, \gamma)}
\end{aligned}
$$

for all $l>d+p+\kappa(|\alpha|+s)$.

Remark 3.3 Two previous lemmas lead to two useful bounds for the operator $H=H_{0}+\mathrm{Op}(b)$ introduced in the beginning with $b \in \mathbf{S}_{\alpha}(w), w=\langle\boldsymbol{\xi}\rangle$.

The estimate (2.8) is an immediate consequence of Lemma 3.1. Indeed, let $\Xi=\mathrm{Op}(w)$. Denote $\tilde{b}(\mathbf{x}, \boldsymbol{\xi})=b(\mathbf{x}, \boldsymbol{\xi})\langle\boldsymbol{\xi}\rangle^{-\alpha}$. Clearly, $\tilde{b}$ satisfies the condition of Lemma 3.1. Thus for any $u \in \mathrm{S}\left(\mathbb{R}^{d}\right)$ and $l>d$ we have

$$
\|\mathrm{Op}(b) u\|=\left\|\mathrm{Op}(\tilde{b}) \Xi^{\alpha} u\right\| \leq C|\tilde{b}|_{l, 0}\left\|\Xi^{\alpha} u\right\| \leq \epsilon\left\|H_{0} u\right\|+C_{\epsilon}\|u\|, \forall \epsilon>0,
$$

as required.

Similarly to (2.8), we can establish a form-bound. Denote

$$
G=\Xi^{-\alpha / 2} B \Xi^{-\alpha / 2} \text {. }
$$

By Lemma 3.2 the symbol $g$ of $G$ satisfies $g \in \mathbf{S}_{0}$ and $|g|_{p, s}^{(0)} \leq C|b|_{l, s}^{(\alpha)}$ with a suitable $l=l(p)$. Thus by Lemma 3.1

$$
|(\mathrm{Op}(b) u, u)|=\left|\left(G \Xi^{\alpha / 2} u, \Xi^{\alpha / 2} u\right)\right| \leq C|b|_{l, 0}^{(\alpha)}\left(\Xi^{\alpha} u, u\right) .
$$

If the symbol $b$ depends on the additional parameter $\sigma$ and $b \in \mathbf{T}_{\alpha, \delta}$, then the same argument yields the bound

$$
|(\mathrm{Op}(b) u, u)|=\left|\left(G \Xi^{\alpha / 2} u, \Xi^{\alpha / 2} u\right)\right| \leq C \sigma^{\delta}|b|_{l, 0,0}^{(\alpha, \delta)}\left(\Xi^{\alpha} u, u\right) .
$$


We are also interested in the estimates for symbols of commutators. For PDO's $A, \Psi_{l}, l=1,2, \ldots, N$, denote

$$
\begin{gathered}
\operatorname{ad}\left(A ; \Psi_{1}, \Psi_{2}, \ldots, \Psi_{N}\right)=i\left[\operatorname{ad}\left(A ; \Psi_{1}, \Psi_{2}, \ldots, \Psi_{N-1}\right), \Psi_{N}\right], \\
\operatorname{ad}(A ; \Psi)=i[A, \Psi], \operatorname{ad}^{N}(A ; \Psi)=\operatorname{ad}(A ; \Psi, \Psi, \ldots, \Psi), \operatorname{ad}^{0}(A ; \Psi)=A .
\end{gathered}
$$

For the sake of convenience we use the notation $\operatorname{ad}\left(a ; \psi_{1}, \psi_{2}, \ldots, \psi_{N}\right)$ and $\operatorname{ad}^{N}(a, \psi)$ for the symbols of multiple commutators. It follows from (3.1) that the Fourier coefficients of the symbol ad $(b, g)$ are given by

$$
\begin{gathered}
\widehat{\operatorname{ad}(b, g)}(\boldsymbol{\chi}, \boldsymbol{\xi})=\frac{i}{\sqrt{\mathrm{d}(\Gamma)}} \sum_{\boldsymbol{\theta}+\boldsymbol{\phi}=\boldsymbol{\chi}}[\hat{b}(\boldsymbol{\theta}, \boldsymbol{\xi}+\boldsymbol{\phi}) \hat{g}(\boldsymbol{\phi}, \boldsymbol{\xi})-\hat{b}(\phi, \boldsymbol{\xi}) \hat{g}(\boldsymbol{\theta}, \boldsymbol{\xi}+\boldsymbol{\phi})], \\
\boldsymbol{\chi} \in \mathbb{Z}^{d}, \boldsymbol{\xi} \in \mathbb{R}^{d} .
\end{gathered}
$$

Lemma 3.4 Let $b \in \mathbf{T}_{\alpha, \delta}$ and $g_{j} \in \mathbf{T}_{\beta_{j}, \epsilon_{j}}, j=1,2, \ldots, N$. Then

$$
\operatorname{ad}\left(b ; g_{1}, \ldots, g_{N}\right) \in \mathbf{T}_{\gamma, \zeta}
$$

with

$$
\gamma=\gamma_{N}=\alpha+\sum_{j=1}^{N}\left(\beta_{j}-1\right), \quad \zeta=\zeta_{N}=\delta+\sum_{j=1}^{N} \epsilon_{j},
$$

and

$$
\mathbf{I} \operatorname{ad}\left(b ; g_{1}, \ldots, g_{N}\right) \mathbf{I}_{l, s, r}^{(\gamma, \zeta)} \leq C_{l, s, r} \mathbf{|} b \mathbf{|}_{p, s+N, r}^{(\alpha, \delta)} \prod_{j=1}^{N} \mid \mathbf{l} g_{j} \mathbf{|}_{p, s+N-j+1, r}^{\left(\beta_{j}, \epsilon_{j}\right)},
$$

with some $p=p\left(l, N, s, \alpha, \beta_{j}\right)$, and a constant $C_{l, s, r}$ independent of $b, g_{j}$.

Proof. As in the proof of Lemma 3.2 we omit the dependence on $\sigma$ from the notation.

The proof is by induction. The claim is trivially true for $N=0$, since by definition $\operatorname{ad}^{o}(b ; g)=b$. Suppose that it is true for $N=M-1, M \geq 1$, i.e. that the symbol of the $M-1$-commutator satisfies

$$
t_{M-1}=\operatorname{ad}\left(b ; g_{1}, \ldots, g_{M-1}\right) \in \mathbf{T}_{\gamma_{M-1}, \zeta_{M-1}},
$$

and the bound (3.5) holds for $N=M-1$. Denote for brevity $\gamma=\gamma_{M-1}, \zeta=$ $\zeta_{M-1}, \beta=\beta_{M}, \epsilon=\epsilon_{M}, g=g_{M}$. According to (3.4) the Fourier transform of the symbol $t_{M}=\operatorname{ad}\left(t_{M-1} ; g\right)$ is:

$$
\begin{gathered}
\hat{t}_{M}(\boldsymbol{\chi}, \boldsymbol{\xi})=\frac{i}{\sqrt{\mathrm{d}(\Gamma)}} \sum_{\boldsymbol{\theta}+\boldsymbol{\phi}=\boldsymbol{\chi}} R(\boldsymbol{\theta}, \boldsymbol{\phi} ; \boldsymbol{\xi}), \\
R(\boldsymbol{\theta}, \boldsymbol{\phi} ; \boldsymbol{\xi})=\hat{t}_{M-1}(\boldsymbol{\theta}, \boldsymbol{\xi}+\boldsymbol{\phi}) \hat{g}(\boldsymbol{\phi}, \boldsymbol{\xi})-\hat{t}_{M-1}(\boldsymbol{\theta}, \boldsymbol{\xi}) \hat{g}(\boldsymbol{\phi}, \boldsymbol{\xi}+\boldsymbol{\theta}) .
\end{gathered}
$$


Rewrite the last formula:

$$
\begin{aligned}
R(\boldsymbol{\theta}, \boldsymbol{\phi} ; \boldsymbol{\xi})=\left[\hat{t}_{M-1}(\boldsymbol{\theta}, \boldsymbol{\xi}+\boldsymbol{\phi})-\hat{t}_{M-1}(\boldsymbol{\theta}, \boldsymbol{\xi})\right] \hat{g}(\boldsymbol{\phi}, \boldsymbol{\xi}) \\
+\hat{t}_{M-1}(\boldsymbol{\theta}, \boldsymbol{\xi})[\hat{g}(\boldsymbol{\phi}, \boldsymbol{\xi})-\hat{g}(\boldsymbol{\phi}, \boldsymbol{\xi}+\boldsymbol{\theta})] .
\end{aligned}
$$

By (2.4) and (2.7)

$$
\begin{aligned}
& \left|\partial_{\sigma}^{n} \mathbf{D}_{\boldsymbol{\xi}}^{\mathbf{s}} R(\boldsymbol{\theta}, \boldsymbol{\phi} ; \boldsymbol{\xi})\right| \leq \\
& \leq C_{p, s, n} \sigma^{-n+\zeta+\epsilon} w(\boldsymbol{\xi})^{\gamma+\beta-1-s}\left(\left.\left|t_{M-1} \mathbf{|}_{p, s+1, n}^{(\gamma, \zeta)}\right| g\right|_{p, s, n} ^{(\beta, \epsilon)}\langle\boldsymbol{\theta}\rangle^{-p}\langle\boldsymbol{\phi}\rangle^{-p+\kappa(|\gamma|+2+s)}\right. \\
& \left.\quad+\left|t_{M-1}\right|_{p, s, n}^{(\gamma, \zeta)}|g|_{p, s+1, n}^{(\beta, \epsilon)}\langle\boldsymbol{\theta}\rangle^{-p+\kappa(|\beta|+2+s)}\langle\boldsymbol{\phi}\rangle^{-p}\right), \quad \forall p \geq 0 .
\end{aligned}
$$

Consequently, for sufficiently large $u=u(l)$ we have

$$
\begin{aligned}
\mid t_{M} \mathbf{|}_{l, s, r}^{(\gamma+\beta-1, \zeta+\epsilon)} & \leq C\left(\left|t_{M-1}\right|_{u, s+1, r}^{(\gamma, \zeta)}|g|_{u, s, r}^{(\beta, \epsilon)}+\left|t_{M-1}\right|_{u, s, r}^{(\gamma, \zeta)}|g|_{u, s+1, r}^{(\beta, \epsilon)}\right) \\
& \leq C\left|t_{M-1}\right|_{u, s+1, r}^{(\gamma, \zeta)}|g|_{u, s+1, r}^{(\beta, \epsilon)} .
\end{aligned}
$$

This means that $t_{M} \in \mathbf{T}_{\gamma+\beta-1, \zeta+\epsilon}$, and, in view of the bound (3.5) for $t_{M-1}$, the corresponding norm of $t_{M}$ is estimated by:

$$
C|b|_{p, s+M, r}^{(\alpha, \delta)} \prod_{j=1}^{M-1}\left|g_{j} \mathbf{|}_{p, s+M-j+1, r}^{\left(\beta_{j}, \epsilon_{j}\right)}\right| g_{M} \mathbf{|}_{u, s+1, r}^{\left(\beta_{M}, \epsilon_{M}\right)},
$$

with some $p$, which yields the bound (3.5) for $N=M$, since $\gamma_{M}=\gamma_{M-1}+$ $\beta_{M}-1$ and $\zeta_{M}=\zeta_{M-1}+\epsilon_{M}$.

\subsection{One-dimensional case. Partition of the perturbation}

So far we have worked with arbitrary dimensions $d \geq 1$. Now we have to narrow down to $d=1$. Also, from now on the weight in the definition of classes $\mathbf{S}_{\alpha}, \mathbf{T}_{\alpha, \delta}$ is assumed to be $w(\xi)=\langle\xi\rangle$. The symbols $b \in \mathbf{T}_{\alpha, \delta}$ are assumed to depend on the parameter $\rho \geq 1$, but sometimes we omit the dependence from the notation. Recall the assumption $\Gamma=2 \pi \mathbb{Z}$ as well.

Let $\Upsilon \in \mathrm{C}_{0}^{\infty}(\mathbb{R})$ be a non-negative function such that

$$
0 \leq \Upsilon \leq 1, \quad \Upsilon(t)=\left\{\begin{array}{l}
1,|t| \leq 1 / 4 \\
0,|t| \geq 1 / 2
\end{array}\right.
$$

Assume also for convenience that $\Upsilon$ is even, i.e. $\Upsilon(t)=\Upsilon(-t)$. Set for $L \geq 1$ and $\theta \in \mathbb{Z}$

$$
\begin{cases}\zeta_{\theta}(\xi ; L)= & \Upsilon\left(\frac{\xi+\theta / 2}{L}\right), \\ \varphi_{\theta}(\xi ; L)= & 1-\zeta_{\theta}(\xi ; L) .\end{cases}
$$


Point out that

$$
\varphi_{\theta}(\xi ; L)=\varphi_{-\theta}(\xi+\theta ; L), \quad \zeta_{\theta}(\xi ; L)=\zeta_{-\theta}(\xi+\theta ; L) .
$$

Note that the functions $\varphi_{\theta}(\xi ; \rho)$ and $\zeta_{\theta}(\xi ; \rho)$, viewed as symbols, belong to the class $\mathbf{T}_{0,0}$. Indeed, suppose that $|\theta| \leq \rho / 8$. Then on the support of $\partial_{\xi} \varphi_{\theta}$ and $\partial_{\xi} \zeta_{\theta}$ one has $3 \rho / 16 \leq|\xi| \leq 9 \rho / 16$, and thus

$$
\left|\partial_{\rho}^{n} \partial_{\xi}^{s} \varphi_{\theta}(\xi ; \rho)\right|+\left|\partial_{\rho}^{n} \partial_{\xi}^{s} \zeta_{\theta}(\xi ; \rho)\right| \leq C_{s} \rho^{-s-n} \leq \tilde{C}_{s}\langle\xi\rangle^{-s} \rho^{-n},|\theta| \leq \rho / 8 .
$$

Let $b \in \mathbf{T}_{\alpha, \delta}=\mathbf{T}_{\alpha, \delta}(\langle\xi\rangle ; 1)$ be a symbol depending on the parameter $\rho \geq 1$. Using $\varphi_{\theta}, \zeta_{\theta}$, we introduce for $b$ five new symbols $b^{\uparrow}, b^{o}, b^{\sharp}, b^{\natural}, b^{b}$ from the class $\mathbf{T}_{\alpha}(\langle\xi\rangle ; 1)$ in the following way:

$$
\begin{gathered}
b^{\uparrow}(x, \xi ; \rho)=\frac{1}{\sqrt{2 \pi}} \sum_{\theta}\left(1-\Upsilon\left(4 \theta \rho^{-1}\right)\right) \hat{b}(\theta, \xi ; \rho) e^{i \theta x}, \\
b^{\sharp}(x, \xi ; \rho)=\frac{1}{\sqrt{2 \pi}} \sum_{\theta \neq 0} \hat{b}(\theta, \xi ; \rho) \varphi_{\theta}(\xi ; 16 \rho) \Upsilon\left(4 \theta \rho^{-1}\right) e^{i \theta x}, \\
b^{\natural}(x, \xi ; \rho)=\frac{1}{\sqrt{2 \pi}} \sum_{\theta \neq 0} \hat{b}(\theta, \xi ; \rho) \varphi_{\theta}(\xi ; \rho) \zeta_{\theta}(\xi ; 16 \rho) \Upsilon\left(4 \theta \rho^{-1}\right) e^{i \theta x}, \\
b^{b}(x, \xi ; \rho)=\frac{1}{\sqrt{2 \pi}} \sum_{\theta \neq 0} \hat{b}(\theta, \xi ; \rho) \zeta_{\theta}(\xi ; \rho) \Upsilon\left(4 \theta \rho^{-1}\right) e^{i \theta x}, \\
b^{o}(x, \xi ; \rho)=b^{o}(\xi ; \rho)=\frac{1}{\sqrt{2 \pi}} \hat{b}(0, \xi ; \rho) .
\end{gathered}
$$

The corresponding operators are denoted by

$$
B^{\uparrow}=\mathrm{Op}\left(b^{\uparrow}\right), B^{\sharp}=\mathrm{Op}\left(b^{\sharp}\right), B^{\natural}=\mathrm{Op}\left(b^{\natural}\right), B^{b}=\mathrm{Op}\left(b^{b}\right), B^{o}=\mathrm{Op}\left(b^{o}\right) .
$$

By definition of $\Upsilon$

$$
b=b^{o}+b^{b}+b^{\natural}+b^{\sharp}+b^{\uparrow} .
$$

The role of each of these operator is easy to explain. The symbol $b^{\uparrow}$ contains only Fourier coefficients with $|\theta| \geq \rho / 16$, and the remaining symbols contain the Fourier coefficients with $|\theta| \leq \rho / 8$. The symbol $b^{b}$ is supported in the region where $\xi$ is small compared to $\rho$, and thus, as it will be shown later, it does not contribute to the asymptotics of the density of states $D\left(\rho^{m}\right)$. The symbol $b^{\sharp}$, on the contrary, contains a cut-off on the "large" values of $\xi$ and hence does not affect $D\left(\rho^{m}\right)$ either. The only significant components of $b$ are the symbols $b^{\natural}$ and $b^{o}$. The symbol $b^{o}$ will remain as it is, and the symbol $b^{\natural}$ will be transformed in the next Section to another symbol, independent of $x$. 
Often we combine $B^{\mathrm{b}}, B^{\sharp}$ and $B^{\uparrow}$ : for instance $B^{\mathrm{b}, \sharp}=B^{\mathrm{b}}+B^{\sharp}, B^{\mathrm{b}, \sharp, \uparrow}=$ $B^{b, \sharp}+B^{\uparrow}$. A similar convention applies to the symbols. In view of (3.9) under the condition $b \in \mathbf{T}_{\alpha, \delta}$ the above symbols belong to class $\mathbf{T}_{\alpha, \delta}$ and the following bounds hold:

$$
\left|b^{b}\right|_{l, s, r}^{(\alpha, \delta)}+\left|b^{\natural}\right|_{l, s, r}^{(\alpha, d)}+\left|b^{\sharp}\right|_{l, s, r}^{(\alpha, \delta)}+\left|b^{o}\right|_{l, s, r}^{(\alpha, \delta)}+\left|b^{\uparrow}\right|_{l, s, r}^{(\alpha, \delta)} \leq C_{l, s, r}|b|_{l, s, r}^{(\alpha, \delta)},
$$

see (2.6) for definition of $\mathbf{I} \cdot \mathbf{I}_{l, s, r}^{(\alpha)}$. To check that the introduced operations preserve symmetry, calculate using (3.8)

$$
\begin{aligned}
\overline{\hat{b}^{b}(-\theta, \xi+\theta)} & =\overline{\hat{b}(-\theta, \xi+\theta)} \zeta_{-\theta}(\xi+\theta ; \rho) \Upsilon\left(4 \theta \rho^{-1}\right) \\
& =\hat{b}(\theta, \xi) \zeta_{\theta}(\xi ; \rho) \Upsilon\left(4 \theta \rho^{-1}\right)=\hat{b}^{b}(\theta, \xi)
\end{aligned}
$$

Therefore, by (2.5) the operator $B^{b}$ is symmetric if so is $B$. Similarly for $B^{\sharp}, B^{\natural}$ and $B^{\uparrow}$.

Let us list some other elementary properties of the introduced operators. In the Lemma below we use the projection $\mathcal{P}(\mathcal{C}), \mathcal{C} \subset \mathbb{R}$ whose definition was given before Proposition 2.1.

Lemma 3.5 Let $b \in \mathbf{T}_{\alpha, \delta}(w ; 1), w=\langle\xi\rangle$, with some $\alpha, \delta \in \mathbb{R}$. Denote for some $R \geq 0$

$$
\Omega_{R}=\{\xi \in \mathbb{R}:|\xi| \geq R\}, \quad \Lambda_{R}=\mathbb{R} \backslash \Omega_{R} .
$$

Then the following statements hold:

(i) The operator $\mathrm{Op}\left(b^{b}\right)$ is bounded and

$$
\left\|\mathrm{Op}\left(b^{b}\right)\right\| \leq C_{l}|b|_{l, 0,0}^{(\alpha, \delta)} \rho^{\alpha_{1}+\delta},
$$

with $\alpha_{1}=\max (\alpha, 0)$. Moreover, for any $R \geq 11 \rho / 16$

$$
\mathcal{P}\left(\Omega_{R}\right) \operatorname{Op}\left(b^{b}\right)=\operatorname{Op}\left(b^{b}\right) \mathcal{P}\left(\Omega_{R}\right)=0 .
$$

(ii) For any $\beta \in \mathbb{R}$ one has $b^{\natural} \in \mathbf{T}_{\beta, \alpha-\beta+\delta}$ and

$$
\left|b^{\natural}\right|_{l, s, r}^{(\beta, \alpha-\beta+\delta)} \leq C|b|_{l, s, r}^{(\alpha, \delta)},
$$

with a constant $C$ depending only on $l, s, r, \alpha, \beta$. In particular, the operator $B^{\natural}$ is bounded and

$$
\left\|B^{\natural}\right\| \leq C_{l}|b|_{l, 0,0}^{(\alpha, \delta)} \rho^{\alpha+\delta},
$$

for any $l>1$. 
(iii) Let $R \leq 3 \rho$. Then

$$
\mathcal{P}\left(\Lambda_{R}\right) B^{\sharp}=B^{\sharp} \mathcal{P}\left(\Lambda_{R}\right)=0 .
$$

(iv) If $R \leq C \rho$, then

$$
\left\|\mathcal{P}\left(\Lambda_{R}\right) B^{\uparrow}\right\|+\left\|B^{\uparrow} \mathcal{P}\left(\Lambda_{R}\right)\right\| \leq C_{l, p}|b|_{l, 0,0}^{(\alpha, \delta)} \rho^{\delta+p-l},
$$

for some fixed $p$ and any sufficiently big $l \geq p$.

Proof. Proof of (i). By (2.7)

$$
|\hat{b}(\theta, \xi ; \rho)| \leq|b|_{l, 0,0}^{(\alpha, \delta)} \rho^{\delta}\langle\theta\rangle^{-l}\langle\xi\rangle^{\alpha}
$$

for any $l>0$. In each term in the r.h.s. of (3.13) the value $|\xi|$ does not exceed $\rho / 2+|\theta / 2| \leq 9 \rho / 16$, so that

$$
\left|\hat{b}^{b}(\theta, \xi ; \rho)\right| \leq C \mid b \mathbf{|}_{l, 0,0}^{(\alpha, \delta)} \rho^{\alpha_{1}+\delta}\langle\theta\rangle^{-l}, \forall l>0,
$$

with $\alpha_{1}=\max \{0, \alpha\}$. By Lemma 3.1 this implies the sought bound for the norm $\left\|\mathrm{Op}\left(b^{b}\right)\right\|$.

Since the value of $|\xi|$ on the support of $b^{b}(x, \cdot ; \rho)$ is less than $9 \rho / 16$, the second part of statement (i) follows from the definition (3.13) by inspection.

Proof of (ii). By (2.7) and (3.9)

$$
\left|\partial_{\rho}^{n} \partial_{\xi}^{s} \hat{b}^{\natural}(\theta, \xi ; \rho)\right| \leq C_{p, s, n} \rho^{\delta-n}|b|_{p, s, n}^{(\alpha, \delta)}\langle\xi\rangle^{\beta-s}\langle\xi\rangle^{\alpha-\beta}|\theta|^{-p} .
$$

Since $3 \rho / 16 \leq|\xi| \leq 9 \rho$ on the support of $b^{\natural}(x, \cdot ; \rho)$, we have

$$
\left|\partial_{\rho}^{n} \partial_{\xi}^{s} \hat{b}^{\natural}(\theta, \xi ; \rho)\right| \leq C_{p, s, n} \rho^{\delta+\alpha-\beta-n}|b|_{p, s, n}^{(\alpha, \delta)}\langle\xi\rangle^{\beta-s}|\theta|^{-p} .
$$

This means that $b^{\natural} \in \mathbf{T}_{\beta, \delta+\alpha-\beta}$ and (3.17) holds. The bound for the norm follows from (3.17) and Lemma 3.1.

Proof of (iii) is similar to (i). Indeed, the value of $|\xi|$ on the support of $b^{\sharp}(x, \cdot ; \rho)$ is greater than $4 \rho-|\theta| / 2 \geq 63 \rho / 16$, so that the required result follows by inspection.

Proof of (iv). By definition of the function $\Upsilon$ the sum (3.10) contains only $\theta:|\theta| \geq \rho / 16$. Thus, in view of (2.6) and (3.15), for any $l \geq p$ we have

$$
\left|\partial_{\rho}^{n} \partial_{\xi}^{s} \hat{b}^{\uparrow}(\theta, \xi ; \rho)\right| \leq \rho^{\delta-n}|b|_{p, s, n}^{(\alpha, \delta)}\langle\xi\rangle^{\alpha-s}|\theta|^{-l} \leq C \rho^{\delta-n+p-l}|b|_{p, s, n}^{(\alpha, \delta)}\langle\xi\rangle^{\alpha-s}|\theta|^{-p},
$$

so that

$$
\mathbf{|} b^{\uparrow} \mathbf{I}_{p, s, r}^{(\alpha, \delta+p-l)} \leq C \mid b^{\uparrow} \mathbf{I}_{l, s, r}^{(\alpha, \delta)}
$$


Instead of $\mathcal{P}\left(\Lambda_{R}\right)$ consider a "smoothed out projection" $E_{R}=\operatorname{Op}\left(\Upsilon_{R}\right)$, $U_{R}(\xi)=\Upsilon\left(\xi(4 R)^{-1}\right)$. By definition of $\Upsilon$ we have $\mathcal{P}\left(\Lambda_{R}\right)=\mathcal{P}\left(\Lambda_{R}\right) E_{R}$. Also, $\Upsilon_{R} \in \mathbf{T}_{\beta, 0}$ for any $\beta \in \mathbb{R}$ and in particular

$$
\mathbf{I} \Upsilon_{R} \mathbf{I}_{l, s, r}^{(-\alpha, 0)} \leq C_{l, s} R^{\alpha_{1}}, \alpha_{1}=\max \{0, \alpha\}
$$

According to Lemma 3.2 the symbol $t$ of the product $E_{R} \mathrm{Op}\left(b^{\uparrow}\right)$ belongs to the class $\mathbf{T}_{0, \delta+p-l}$ and

$$
\begin{aligned}
\left.|t|\right|_{r, 0,0} ^{(0, \delta+p-l)} & \leq C\left|b^{\uparrow}\right|_{p, 0,0}^{(\alpha, \delta+p-l)} \mid \Upsilon_{R} \mathbf{|}_{p, 0}^{(-\alpha, 0)} \\
& \leq C_{l}^{\prime}|b|_{l, 0,0}^{(\alpha, \delta)} \rho^{p-l+\alpha_{1}},
\end{aligned}
$$

for some $p=p(r)$ and arbitrary $l \geq p$. By Lemma 3.1 this proves the bound (3.18) for $\mathcal{P}\left(\Lambda_{R}\right) \operatorname{Op}\left(b^{\uparrow}\right)$. The same argument leads to the same bound for $\operatorname{Op}\left(b^{\uparrow}\right) \mathcal{P}\left(\Lambda_{R}\right)$.

\section{A "gauge transformation"}

In this and all the subsequent sections we assume that $\mathbf{S}_{\alpha}=\mathbf{S}_{\alpha}(w), \mathbf{T}_{\alpha, \delta}=$ $\mathbf{T}_{\alpha, \delta}(w ; 1)$ with $w(\xi)=\langle\xi\rangle$. Symbols from $\mathbf{T}_{\alpha, \delta}$ are supposed to depend on the parameter $\rho \geq 1$.

\subsection{Preparation}

Suppose that $b \in \mathbf{T}_{\alpha, \delta}$ with some $\alpha<m$ and $\delta \in \mathbb{R}$. Our strategy will be to find a unitary operator which reduces $H=H_{0}+\mathrm{Op}(b)$ to another PDO, whose symbol, up to some controllable small errors, depends only on $\xi$. The sought unitary operator will be constructed in the form $U=e^{i \Psi}$ with a suitable bounded self-adjoint $\Gamma$-periodic PDO $\Psi$. This is why we sometimes call it a "gauge transformation". It is useful to consider $e^{i \Psi}$ as an element of the group

$$
U(t)=\exp \{i \Psi t\}, \quad \forall t \in \mathbb{R} .
$$

We assume that the operator $\operatorname{ad}\left(H_{0}, \Psi\right)$ is bounded, so that

$$
U(t) D\left(H_{0}\right)=D\left(H_{0}\right) .
$$

This assumption will be justified later on. Let us express the operator

$$
A_{t}=U(-t) H U(t)
$$

via its (weak) derivative w.r.t. $t$ :

$$
A_{t}=H+\int_{0}^{t} U\left(-t^{\prime}\right) \operatorname{ad}(H ; \Psi) U\left(t^{\prime}\right) d t^{\prime} .
$$


By induction it is easy to show that

$$
A_{1}=H+\sum_{j=1}^{M} \frac{1}{j !} \operatorname{ad}^{j}(H ; \Psi)+R_{M+1}^{(1)}
$$

$$
R_{M+1}^{(1)}=\int_{0}^{1} d t_{1} \int_{0}^{t_{1}} d t_{2} \ldots \int_{0}^{t_{M}} U\left(-t_{M+1}\right) \operatorname{ad}^{M+1}(H ; \Psi) U\left(t_{M+1}\right) d t_{M+1} .
$$

The operator $\Psi$ is sought in the form

$$
\Psi=\sum_{k=1}^{M} \Psi_{k}, \Psi_{k}=\mathrm{Op}\left(\psi_{k}\right)
$$

with symbols $\psi_{k}$ from some suitable class $\mathbf{T}_{\beta, \epsilon}, \beta=\beta_{k}, \epsilon=\epsilon_{k}$ to be specified later on. Substitute this formula in (4.1) and rewrite, regrouping the terms:

$A_{1}=H_{0}+B+\sum_{j=1}^{M} \frac{1}{j !} \sum_{l=j}^{M} \sum_{k_{1}+k_{2}+\cdots+k_{j}=l} \operatorname{ad}\left(H ; \Psi_{k_{1}}, \Psi_{k_{2}}, \ldots, \Psi_{k_{j}}\right)+R_{M+1}^{(1)}+R_{M+1}^{(2)}$,

$$
R_{M+1}^{(2)}=\sum_{j=1}^{M} \frac{1}{j !} \sum_{k_{1}+k_{2}+\cdots+k_{j} \geq M+1} \operatorname{ad}\left(H ; \Psi_{k_{1}}, \Psi_{k_{2}}, \ldots, \Psi_{k_{j}}\right) .
$$

Rewrite:

$$
\begin{aligned}
A_{1}= & H_{0}+B+\sum_{l=1}^{M} \operatorname{ad}\left(H_{0} ; \Psi_{l}\right)+\sum_{j=2}^{M} \frac{1}{j !} \sum_{l=j}^{M} \sum_{k_{1}+k_{2}+\cdots+k_{j}=l} \operatorname{ad}\left(H_{0} ; \Psi_{k_{1}}, \Psi_{k_{2}}, \ldots, \Psi_{k_{j}}\right) \\
& +\sum_{j=1}^{M} \frac{1}{j !} \sum_{l=j}^{M} \sum_{k_{1}+k_{2}+\cdots+k_{j}=l} \operatorname{ad}\left(B ; \Psi_{k_{1}}, \Psi_{k_{2}}, \ldots, \Psi_{k_{j}}\right)+R_{M+1}^{(1)}+R_{M+1}^{(2)} .
\end{aligned}
$$

Switch the summation signs:

$$
\begin{aligned}
A_{1} & =H_{0}+B+\sum_{l=1}^{M} \operatorname{ad}\left(H_{0} ; \Psi_{l}\right)+\sum_{l=2}^{M} \sum_{j=2}^{l} \frac{1}{j !} \sum_{k_{1}+k_{2}+\cdots+k_{j}=l} \operatorname{ad}\left(H_{0} ; \Psi_{k_{1}}, \Psi_{k_{2}}, \ldots, \Psi_{k_{j}}\right) \\
& +\sum_{l=2}^{M+1} \sum_{j=1}^{l-1} \frac{1}{j !} \sum_{k_{1}+k_{2}+\cdots+k_{j}=l-1} \operatorname{ad}\left(B ; \Psi_{k_{1}}, \Psi_{k_{2}}, \ldots, \Psi_{k_{j}}\right)+R_{M+1}^{(1)}+R_{M+1}^{(2)} .
\end{aligned}
$$

Introduce the notation

$$
\begin{gathered}
B_{1}=B \\
B_{l}=\sum_{j=1}^{l-1} \frac{1}{j !} \sum_{k_{1}+k_{2}+\cdots+k_{j}=l-1} \operatorname{ad}\left(B ; \Psi_{k_{1}}, \Psi_{k_{2}}, \ldots, \Psi_{k_{j}}\right), l \geq 2, \\
T_{l}=\sum_{j=2}^{l} \frac{1}{j !} \sum_{k_{1}+k_{2}+\cdots+k_{j}=l} \operatorname{ad}\left(H_{0} ; \Psi_{k_{1}}, \Psi_{k_{2}}, \ldots, \Psi_{k_{j}}\right), l \geq 2 .
\end{gathered}
$$


We emphasise that the operators $B_{l}$ and $T_{l}$ depend only on $\Psi_{1}, \Psi_{2}, \ldots, \Psi_{l-1}$. Let us make one more rearrangement:

$$
\begin{gathered}
A_{1}=H_{0}+B+\sum_{l=1}^{M} \operatorname{ad}\left(H_{0}, \Psi_{l}\right)+\sum_{l=2}^{M} B_{l}+\sum_{l=2}^{M} T_{l}+R_{M+1}, \\
R_{M+1}=B_{M+1}+R_{M+1}^{(1)}+R_{M+1}^{(2)} .
\end{gathered}
$$

Now we can specify our algorithm for finding $\Psi_{k}$ 's. The symbols $\psi_{k}$ will be found from the following system of commutator equations:

$$
\begin{gathered}
\operatorname{ad}\left(H_{0} ; \Psi_{1}\right)+B_{1}^{\natural}=0, \\
\operatorname{ad}\left(H_{0} ; \Psi_{l}\right)+B_{l}^{\natural}+T_{l}^{\natural}=0, l \geq 2,
\end{gathered}
$$

and hence

$$
\begin{gathered}
A_{1}=A_{0}+L_{M}^{b, \sharp, \uparrow}(\Psi)+R_{M+1}, \\
L_{M}=\sum_{l=1}^{M} B_{l}+\sum_{l=2}^{M} T_{l}, \\
A_{0}=H_{0}+\sum_{l=1}^{M} B_{l}^{o}+\sum_{l=2}^{M} T_{l}^{o} .
\end{gathered}
$$

Below we denote by $l_{M}$ the symbol of the PDO $L_{M}$. Recall that by Lemma 3.5(ii), the operators $B_{l}^{\natural}, T_{l}^{\natural}$ are bounded, and therefore, in view of (4.7), (4.8), so is the commutator ad $\left(H_{0} ; \Psi\right)$. This justifies the assumption made in the beginning of the formal calculations in this Section.

\subsection{Commutator equations}

Before proceeding to the study of the commutator equations (4.7), (4.8) note a few bounds for the symbol

$$
\tau_{\theta}(\xi)=h_{0}(\xi+\theta)-h_{0}(\xi) .
$$

Assuming that $|\xi| \geq 3|\theta| / 2$, we obtain that

$$
\begin{gathered}
c|\theta|\langle\xi\rangle^{m-1} \leq\left|\tau_{\theta}(\xi)\right| \leq C|\theta|\langle\xi\rangle^{m-1}, \\
\left|\partial_{\xi}^{s} \tau_{\theta}(\xi)\right| \leq C_{s}|\theta|\langle\xi\rangle^{m-1-s} .
\end{gathered}
$$

Moreover,

$$
\left|\partial_{\xi}^{s} \tau_{\theta}^{-1}\right| \leq C_{s}|\theta|^{-1}\langle\xi\rangle^{-m+1-s},|\xi| \geq 3|\theta| / 2>0 .
$$

Note that the condition $|\xi| \geq 3|\theta| / 2$ is satisfied for $\xi$ on the support of $\varphi_{\theta}(\cdot ; \rho)$ for $|\theta| \leq \rho / 8$. These estimates will come in handy in the next lemma. 
Lemma 4.1 Let $A=\mathrm{Op}(a)$ be a symmetric PDO such that $a \in \mathbf{T}_{\alpha, \delta}$. Then the PDO $\Psi$ with the Fourier coefficients of the symbol $\psi(x, \xi ; \rho)$ given by

$$
\left\{\begin{array}{l}
\hat{\psi}(\theta, \xi ; \rho)=i \frac{\hat{a}^{\natural}(\theta, \xi ; \rho)}{\tau_{\theta}(\xi)}, \theta \neq 0, \\
\hat{\psi}(0, \xi ; \rho)=0,
\end{array}\right.
$$

solves the equation

$$
\operatorname{ad}\left(H_{0} ; \Psi\right)+\operatorname{Op}\left(a^{\natural}\right)=0 .
$$

Moreover, the operator $\Psi$ is bounded and self-adjoint, $\psi \in \mathbf{T}_{\beta, \delta+\alpha-\beta-m+1}$ for all $\beta \in \mathbb{R}$, and

$$
\mathbf{|} \psi \mathbf{|}_{l, s, r}^{(\beta, \delta+\alpha-\beta-m+1)} \leq C \mid a \mathbf{|}_{p, s, r}^{(\alpha, \delta)},
$$

with some $p=p(l, s, r)$. The constant $C$ is independent of the parameter $\rho \geq 1$ and the symbol $a$.

Proof. For brevity we omit $\rho$ from the notation. Let $t$ be the symbol of $\operatorname{ad}\left(H_{0} ; \Psi\right)$. The Fourier transform $\hat{t}(\theta, \xi)$ is easy to find using (3.1):

$$
\hat{t}(\theta, \xi)=i\left(h_{0}(\xi+\theta)-h_{0}(\xi)\right) \hat{\psi}(\theta, \xi)=i \tau_{\theta}(\xi) \hat{\psi}(\theta, \xi) .
$$

Therefore the equation (4.14) amounts to

$$
i \tau_{\theta}(\xi) \hat{\psi}(\theta, \xi)=-\hat{a}^{\natural}(\theta, \xi ; \rho)=-\hat{a}(\theta, \xi ; \rho) \varphi_{\theta}(\xi ; \rho) \zeta_{\theta}(\xi ; 16 \rho) \Upsilon\left(4|\theta| \rho^{-1}\right) .
$$

By definition (3.12), a solution $\hat{\psi}$ exists and is given by (4.13). The symbol $\hat{\psi}$ satisfies the condition (2.5), so that $\Psi$ is a symmetric operator. Recall also that by Lemma 3.5(ii)

$$
\left|a^{\natural}\right|_{l, s, r}^{(\beta, \alpha-\beta+\delta)} \leq C|a|_{l, s, r}^{(\alpha, \delta)},
$$

for any $\beta \in \mathbb{R}$, and hence, using (4.12) we get (4.15). The estimate (4.15) with $\beta=0$ and Lemma 3.1 ensure the boundedness of $\Psi$.

Remark 4.2 Let $a$ be as in Lemma 4.1 and consider the commutator $\operatorname{ad}(\mathrm{Op}(g), \Psi)$ with some symmetric symbol $g \in \mathbf{T}_{\gamma, \zeta}$. By (3.4) the symbol $(\operatorname{ad}(g, \psi))^{o}$ is given by the formula

$$
\begin{aligned}
\hat{t}(0, \xi) & =i \frac{1}{(2 \pi)^{1 / 2}} \sum_{\theta}[\hat{g}(-\theta, \xi+\theta) \hat{\psi}(\theta, \xi)-\hat{g}(\theta, \xi) \hat{\psi}(-\theta, \xi+\theta)] \\
& =i \frac{1}{(2 \pi)^{1 / 2}} \sum_{\theta}[\overline{\hat{g}(\theta, \xi)} \hat{\psi}(\theta, \xi)-\hat{g}(\theta, \xi) \overline{\hat{\psi}(\theta, \xi)}] \\
& =-\frac{1}{(2 \pi)^{1 / 2}} \sum_{\theta} \frac{1}{\tau_{\theta}(\xi)}\left[\overline{\hat{g}(\theta, \xi)} \hat{a}^{\natural}(\theta, \xi)+\hat{g}(\theta, \xi) \overline{\hat{a}^{\natural}(\theta, \xi)}\right] .
\end{aligned}
$$


Let us apply Lemma 4.1 to equations (4.7) and (4.8). Recall the notation $\varkappa=m-\alpha$.

Lemma 4.3 Let $b \in \mathbf{T}_{\alpha, \delta}$ be a symmetric symbol, and denote

$$
\zeta_{l}=(\delta-\varkappa) l+1, \quad \epsilon_{l}=(\delta-\varkappa) l+m, l \geq 1 .
$$

Then there exists a sequence of self-adjoint bounded PDO's $\Psi_{l}, l=1,2, \ldots$ with the symbols $\psi_{l} \in \mathbf{T}_{\beta, \zeta_{l}-\beta}$ for any $\beta \in \mathbb{R}$, such that (4.7) and (4.8) hold, and

(i)

$$
\left|\psi_{l}\right|_{r, s, u}^{\left(\beta, \zeta_{l}-\beta\right)} \leq C\left(\left.|| b\right|_{p, n, u} ^{(\alpha, \delta)}\right)^{l}, l \geq 1
$$

(ii) The symbols $b_{l}, t_{l}$ of the corresponding operators $B_{l}, T_{l}$ belong to $\mathbf{T}_{\gamma, \epsilon_{l}-\gamma}$ with arbitrary $\gamma \in \mathbb{R}$, and

$$
\left|b_{l} \mathbf{|}_{r, s, u}^{\left(\gamma, \epsilon_{l}-\gamma\right)}+\right| t_{l} \mathbf{|}_{r, s, u}^{\left(\gamma, \epsilon_{l}-\gamma\right)} \leq C\left(|b|_{p, n, u}^{(\alpha, \delta)}\right)^{l}, l \geq 2
$$

(iii) The symbols $b_{l}^{o}(\xi ; \rho)$ and $t_{l}^{o}(\xi ; \rho)$ belong to the class $\mathcal{T}_{l, \delta}^{m}(0,1)$, and

$$
b_{2}^{o}(\xi ; \rho)+t_{2}^{o}(\xi ; \rho)=-\frac{1}{2 \pi} \sum_{\theta \neq 0} \frac{|\hat{b}(\theta, \xi ; \rho)|^{2}}{\tau_{\theta}(\xi)} \Upsilon\left(4|\theta| \rho^{-1}\right)\left[2-\Upsilon\left(4|\theta| \rho^{-1}\right)\right]
$$

for all $3 \rho / 4 \leq|\xi| \leq 2 \rho$.

The constant $C$ in (4.16) and (4.17) does not depend on b, but depends on $l, r, s, \alpha, \beta, \delta, m, u$. The integer-valued parameters $p, n$ in (4.16) and (4.17) depend on $l, r, s, \alpha, \beta, \delta, m$.

(iv) Suppose that $\delta \leq \varkappa$ and that $\left.||\right|_{l, s, 0} ^{(\alpha, \delta)} \leq C_{l, s}$ for all $l$ and $s$, then for some positive integer $p, n$ the following bound holds:

$$
\left\|R_{M+1}\right\| \leq \tilde{C}\left(||||_{p, n, 0}^{(\alpha, \delta)}\right)^{M+1} \rho^{(\delta-\varkappa)(M+1)+m} .
$$

The constant $\tilde{C}$ depends only on the constants $C_{l, s}$ and the parameters $M, \alpha, m$.

Proof. The existence of $\psi_{1} \in \mathbf{T}_{\beta, \zeta_{1}}$ with the required properties follows from Lemma 4.1. Further proof is by induction as follows.

To make the calculations less cumbersome, throughout the proof we adopt the following notational convention. If two symbols, $\phi_{1}$ and $\phi_{2}$ satisfy the estimate $\left|\phi_{1} \mathbf{l}_{l, s, r}^{(\beta, \chi)} \leq C\right| \phi_{2} \mathbf{|}_{p, n, r}^{(\omega, \gamma)}$ with some $p=p(l, s)$ and $n=n(l, s)$ and all $r \geq 0$, we simply write $\left|\phi_{1}\right|^{(\beta, \chi)} \leq C\left|\phi_{2}\right|^{(\omega, \gamma)}$. 
Suppose that $\psi_{k}$ with $k=1,2, \ldots, K-1$ satisfy (4.16). In order to conclude that $\psi_{K}$ also satisfies (4.16), first we need to check that $b_{K}$ and $t_{K}$ satisfy (4.17).

Step I. Estimates for $b_{l}$. To begin with we prove that all the symbols $b_{l}$ with $l \leq K$, satisfy the estimate (4.17). We first obtain a bound for $\operatorname{ad}\left(b ; \psi_{k_{1}} \psi_{k_{2}}, \ldots, \psi_{k_{j}}\right)$ with $k_{1}+k_{2}+\cdots+k_{j}=l-1$. To this end we use (4.16) with $\beta=(\gamma-\alpha) j^{-1}+1$ and Lemma 3.4 to conclude that

$$
\begin{gathered}
\left|\operatorname{ad}\left(b ; \psi_{k_{1}}, \psi_{k_{2}}, \ldots, \psi_{k_{j}}\right)\right|^{\left(\gamma, \epsilon_{j}-\gamma\right)} \leq C|b|^{(\alpha, \delta)} \prod_{n=1}^{j}\left|\psi_{k_{n}}\right|^{\left(\beta, \zeta_{k_{n}}-\beta\right)} \\
\leq C|b|^{(\alpha, \delta)} \prod_{n=1}^{j}\left(\left.|| b\right|^{(\alpha, \delta)}\right)^{k_{n}}=C\left(\left.|| b\right|^{(\alpha, \delta)}\right)^{l} .
\end{gathered}
$$

This implies that $b_{l}$ satisfies (4.17) for all $l \leq K$.

Step II. Estimates for $t_{l}$. For the symbols $t_{l}$ the proof is by induction. First of all, note that

$$
\operatorname{ad}\left(h_{0} ; \psi_{1}, \psi_{1}\right)=-\operatorname{ad}\left(b^{\natural}, \psi_{1}\right),
$$

so that, by Lemma 3.4, (3.17) and (4.2), for $\beta=\gamma-\alpha+1$ we have:

$$
\left|\operatorname{ad}\left(h_{0} ; \psi_{1}, \psi_{1}\right)\right|^{\left(\gamma, \epsilon_{2}-\gamma\right)} \leq C\left|b^{\natural}\right|^{(\alpha, \delta)}\left|\psi_{1}\right|^{\left(\beta, \zeta_{1}-\beta\right)} \leq C\left(\left.|| b\right|^{(\alpha, \delta)}\right)^{2},
$$

and thus $t_{2}$ satisfies (4.17). Suppose that all $t_{k}$ with $k \leq l-1 \leq K-1$ satisfy (4.17). Then by definition (4.8), Step I of this proof, and (3.15), all $\operatorname{ad}\left(h_{0} ; \psi_{k}\right), k \leq l-1$, satisfy the same bound:

$$
\mid \operatorname{ad}\left(h_{0} ; \psi_{k}\right) \mathbf{|}^{\left(\beta, \epsilon_{k}-\beta\right)} \leq C\left(\mid \boldsymbol{|} b \mathbf{|}^{(\alpha, \delta)}\right)^{k} .
$$

Remembering that the definition (4.5) of $t_{l}$ involves only $\psi_{k}$ with $k \leq l-1$, and applying Lemma 3.4 again, we obtain for $k_{1}+k_{2}+\cdots+k_{j}=l, j \geq 2$ and $\beta=(\gamma-1) j^{-1}+1$ :

$$
\begin{array}{r}
\mathbf{|} \operatorname{ad}\left(h_{0} ; \psi_{k_{1}}, \psi_{k_{2}}, \ldots, \psi_{k_{j}}\right) \mathbf{|}^{\left(\gamma, \epsilon_{l}-\gamma\right)}=\mathbf{|} \operatorname{ad}\left(\operatorname{ad}\left(h_{0} ; \psi_{k_{1}}\right) ; \psi_{k_{2}}, \ldots, \psi_{k_{j}}\right) \mathbf{|}^{\left(\gamma, \epsilon_{l}-\gamma\right)} \\
\leq C\left|\operatorname{ad}\left(h_{0} ; \psi_{k_{1}}\right) \mathbf{|}^{\left(\beta, \epsilon_{k_{1}}-\beta\right)} \prod_{n=2}^{j}\right| \psi_{k_{n}} \mathbf{|}^{\left(\beta, \zeta_{k_{n}}-\beta\right)} \leq C\left(|\boldsymbol{|}| \mathbf{|}^{(\alpha, \delta)}\right)^{l},
\end{array}
$$

which leads to (4.17) for all $t_{l}, l \leq K$.

Step III. To handle $\Psi_{K}$ we use the solution $\Psi$ of the equation (4.14) constructed in Lemma 4.1. Then from definition (4.8), (4.15) and Steps I, II we immediately conclude that $\psi_{K} \in \mathbf{T}_{\beta, \zeta_{K}-\beta}$ with arbitrary $\beta \in \mathbb{R}$ and that

$$
\left|\psi_{K}\right|^{\left(\beta, \zeta_{K}-\beta\right)} \leq C\left(\left.|| b_{K}\right|^{\left(\beta, \epsilon_{K}-\beta\right)}+\left|t_{K}\right|^{\left(\beta, \epsilon_{K}-\beta\right)}\right) \leq C\left(\left.|| b\right|^{(\alpha, \delta)}\right)^{K},
$$

as required. 
Step IV. Proof of (iii). The first statement is an immediate consequence of (4.17) with $\gamma=\epsilon_{l}$ and (3.15).

By (4.4) and by (4.5), (4.7)

$$
B_{2}=\operatorname{ad}\left(B ; \Psi_{1}\right), T_{2}=-\frac{1}{2} \operatorname{ad}\left(B^{\natural} ; \Psi_{1}\right) .
$$

It follows from (4.13) that

$$
\hat{\psi}_{1}(\theta, \xi ; \rho)=i \frac{\hat{b}^{\natural}(\theta, \xi ; \rho)}{\tau_{\theta}(\xi)} .
$$

Remark 4.2 and definition (3.14) lead to the formula

$$
t_{2}^{o}(\xi ; \rho)=\frac{1}{2 \pi} \sum_{\theta \neq 0} \frac{\left|\hat{b}^{\natural}(\theta, \xi ; \rho)\right|^{2}}{\tau_{\theta}(\xi)} .
$$

For all $|\xi| \in[9 \rho / 16,47 \rho / 16]$ and $|\theta| \leq \rho / 8$ we have the bound $\rho / 2 \leq \mid \xi+$ $\theta / 2 \mid \leq 3 \rho$, and therefore, by $(3.12) b^{\natural}(\theta, \xi ; \rho)=b(\theta, \xi)$. Consequently,

$$
t_{2}^{o}(\xi ; \rho)=\frac{1}{2 \pi} \sum_{\theta \neq 0} \frac{|\hat{b}(\theta, \xi ; \rho)|^{2}}{\tau_{\theta}(\xi)}\left[\Upsilon\left(4|\theta| \rho^{-1}\right)\right]^{2} .
$$

Similarly one shows that the symbol $b_{2}^{o}(\xi)$ of $\left(\operatorname{ad}\left(B ; \Psi_{1}\right)\right)^{o}$ satisfies the formula

$$
b_{2}^{o}(\xi ; \rho)=-\frac{1}{\pi} \sum_{\theta \neq 0} \frac{|\hat{b}(\theta, \xi ; \rho)|^{2}}{\tau_{\theta}(\xi)} \Upsilon\left(4|\theta| \rho^{-1}\right),
$$

and hence (4.18) is fulfilled.

Step V. Bounds for the remainder. Until the end of the proof we denote by $|b|^{(\alpha, \delta)}$ the norm $|b|_{r, s, 0}^{(\alpha, \delta)}$ with some suitable $r, s$. The remainder $R_{M+1}$ (see (4.6)) consists of three components. In view of (4.17) with $\gamma=0$, and Lemma 3.1, the norm of $B_{M+1}$ is bounded by

$$
C\left(\left.|| b\right|^{(\alpha, \delta)}\right)^{M+1} \rho^{(\delta-\varkappa)(M+1)+m},
$$

as required.

Consider now $R_{M+1}^{(1)}$ defined in (4.1). Let $\psi=\sum_{l=1}^{M} \psi_{l}$. Since $\delta \leq \varkappa$ and $|b|^{(\alpha, \delta)} \leq C$, we have $\mathbf{T}_{\beta, \zeta_{l}-\beta} \subset \mathbf{T}_{\beta, \zeta_{1}-\beta}$ and hence, in view of (4.2),

$$
\left.\left|\psi_{l} \mathbf{|}^{\left(\beta, \zeta_{1}-\beta\right)} \leq C_{l}\right| b\right|^{(\alpha, \delta)}, l \geq 1, \quad \text { and } \quad|\psi|^{\left(\beta, \zeta_{1}-\beta\right)} \leq C|b|^{(\alpha, \delta)} .
$$

Similarly, by definition (4.10) we have $\left|l_{M}\right|^{\left(\beta, \epsilon_{1}-\beta\right)} \leq C_{l}|b|^{(\alpha, \delta)}$ in view of (4.17). 
It follows from (4.2), (4.7) and (4.8) that $\operatorname{ad}\left(H_{0}, \Psi\right)+L_{M}^{\natural}=0$. Now it follows from (3.15) that

$$
\mathbf{| a d}\left(h_{0}, \psi\right) \mathbf{|}^{\left(\beta, \epsilon_{1}-\beta\right)} \leq\left. C\left|l_{M} \mathbf{|}^{\left(\beta, \epsilon_{1}-\beta\right)} \leq C\right| b\right|^{(\alpha, \delta)} .
$$

Using (4.22) and Lemma 3.4 we conclude that

$$
\left|\operatorname{ad}^{M+1}(h, \psi)\right|^{\left(\gamma, \epsilon_{M+1}-\gamma\right)} \leq C\left(|\boldsymbol{|} b|^{(\alpha, \delta)}\right)^{M+1}
$$

for any $\gamma \in \mathbb{R}$. By Lemma 3.1 this leads to the required estimate for the norm $\left\|R_{M+1}^{(1)}\right\|$.

In the same way the norm of the error $R_{M+1}^{(2)}$ defined in (4.3) can be shown to satisfy the same bound. This completes the proof of (4.19).

Let us now summarise the results of this section in the following Theorem:

Theorem 4.4 Let $b \in \mathbf{T}_{\alpha, \delta}, \alpha<m, \delta \leq \varkappa=m-\alpha$, be a symmetric symbol. Then for any positive integer $M$ there exists a symbol $\psi \in \mathbf{T}_{\beta, \epsilon_{1}-\beta}$, $\forall \beta \in \mathbb{R}$, of the form (4.2) such that

(i) the operator $A_{1}=e^{-i \Psi} H e^{i \Psi}$ has the form (4.9), and

(ii) the conclusions of Lemma 4.3 hold for the symbols $\psi_{l}, b_{l}, t_{l}$ and the remainder $R_{M+1}$.

This theorem reduces the operator $H$ to a form convenient for further calculation of the density of states. The next step is to carry out such a calculation for the operator $A_{0}$ with constant coefficients. After that it will be shown that the remaining components of $A_{1}$, that is $L^{\uparrow}, L^{b}$ and $L^{\sharp}$, do not affect the asymptotics.

\section{Density of states}

\subsection{Operator $A_{0}$}

We begin our study of the density of states with the operator $A_{0}$ defined in (4.11). According to Lemma 4.3(iii) we have $b_{l}^{o}, t_{l}^{o} \in \mathcal{T}_{l, \delta}^{m}(0,1)$, so that the symbol $a_{0}(\xi ; \rho)$ of $A_{0}$ can be written as

$$
a_{0}(\xi ; \rho)=|\xi|^{m}+\sum_{j=1}^{M} \mu_{j}(\xi ; \rho), \rho \geq 1,
$$

where $\mu_{j} \in \mathcal{T}_{j, \delta}^{m}(0,1)$ uniformly in the symbol $b$ and $\mu_{1}=b^{o}, \mu_{j}=b_{j}^{o}+t_{j}^{o}$, $j \geq 2$. Since $A_{0}$ has a symbol independent of $x$, in order to find its density of states or $\hat{N}\left(\rho^{m} ; 2 \pi n\right), n \in \mathbb{Z}$, we can immediately use Proposition 2.1. To this end we need to identify the set where $a_{0}(\xi ; \rho)<\rho^{m}$. This is the objective of the next lemma. 
In this lemma we consider a more general symbol which is given by an asymptotic series:

$$
a(\xi ; \rho)=|\xi|^{m}+\sum_{j=1}^{\infty} \mu_{j}(\xi ; \rho), \mu_{j} \in \mathcal{T}_{j, \delta}^{m}\left(\xi_{0}, 1\right)
$$

with some $\xi_{0}>0$. Define a sequence of functions $\omega_{j}=\omega_{j}(\eta ; \rho), j=1,2, \ldots$ by the following recursion relations:

$$
\begin{gathered}
m|\eta|^{m} \eta^{-1} \omega_{1}+\mu_{1}=0, \\
\omega_{l}=-\frac{\eta}{m|\eta|^{m}} \mu_{l}-\sum_{s=2}^{l} \frac{(m-1) \cdots(m-s+1)}{s !} \eta^{-s+1} \sum_{p_{1}+p_{2}+\cdots+p_{s}=l} \omega_{p_{1}} \cdots \omega_{p_{s}} \\
\text { (5.3) } \quad-\frac{\eta}{m|\eta|^{m}} \sum_{s=1}^{l-1} \frac{1}{s !} \sum_{j+p_{1}+p_{2}+\cdots+p_{s}=l} \partial^{s} \mu_{j}(\eta) \omega_{p_{1}} \cdots \omega_{p_{s}}, l \geq 2 .
\end{gathered}
$$

One can easily compute the first two functions $\omega_{j}$ :

$$
\begin{aligned}
& \omega_{1}=-\frac{1}{m} \eta|\eta|^{-m} \mu_{1}, \\
& \omega_{2}=-\frac{1}{m} \eta|\eta|^{-m} \mu_{2}-\frac{m-1}{2 m^{2}} \eta^{-2 m+1} \mu_{1}^{2}+\frac{1}{2 m^{2}} \eta^{2-2 m}\left(\mu_{1}^{2}\right)^{\prime} .
\end{aligned}
$$

It is clear from (5.4) that $\omega_{1} \in \mathcal{T}_{1, \delta}^{1}\left(\xi_{0}, 1\right)$ uniformly in the series for $a$. This property implies the inclusion $\omega_{j} \in \mathcal{T}_{j, \delta}^{1}\left(\xi_{0}, 1\right)$ for all $j \geq 2$, also uniformly in a. Indeed, suppose that $\omega_{j} \in \mathcal{T}_{j, \delta}^{1}\left(\xi_{0}, 1\right)$ for all $j \leq J$. Since the r.h.s. of the equality (5.3) depends only on $\omega_{j}, j \leq l-1$, it is a matter of routine to check that the r.h.s. in the equality (5.3) with $l=J+1$ belongs to $\mathcal{T}_{J+1, \delta}^{1}\left(\xi_{0}, 1\right)$. Thus $\omega_{J+1} \in \mathcal{T}_{J+1, \delta}^{1}\left(\xi_{0}, 1\right)$.

Recall that in the formula (5.1) the symbols $\mu_{j}$ belong to $\mathcal{T}_{j, \delta}^{m}(0,1)$, so that in this case $\omega_{j} \in \mathcal{T}_{j, \delta}^{1}\left(\xi_{0}, 1\right)$ for any $\xi_{0}>0$.

Lemma 5.1 Let a be as defined in (5.2) and let $\delta<\varkappa$. Then there exists an $\eta_{0} \geq \xi_{0}$, uniform in the series a, such that for all $\eta:|\eta| \geq \eta_{0}$ the equation $a(\xi ; \rho)=|\eta|^{m}$ has exactly one root $\xi(\eta ; \rho)$ having the same sign as $\eta$. The function $\xi(\eta ; \rho)$ can be represented as an asymptotic series

$$
\xi(\eta ; \rho)=\eta+\sum_{j=1}^{\infty} \omega_{j}(\eta ; \rho),
$$

with functions $\omega_{j} \in \mathcal{T}_{j, \delta}^{1}\left(\xi_{0}, 1\right)$ that are defined by (5.3). The first two terms have the form (5.4) and (5.5). The asymptotics (5.6) are uniform with respect to the series for $a$.

Note that the existence of the functions $\xi(\eta ; \rho)$ is claimed in the region $|\eta| \geq \eta_{0}$ which is generally speaking smaller than the region where the functions $\omega_{j}$ are defined. 
Proof. To avoid unnecessary repetitions, throughout the proof we always tacitly assume that the inclusion $f \in \mathcal{T}_{j, \delta}^{\nu}(\cdot, \cdot)$ for some function $f$ means that $f$ belongs to $\mathcal{T}_{j, \delta}^{\nu}(\cdot, \cdot)$ uniformly in the series $a$. Also, the symbol $u_{j}^{(\nu)}$ denotes a function which satisfies the bound

$$
\left|u_{j}^{(\nu)}(\xi ; \rho)\right| \leq C_{j}\langle\xi\rangle^{j(\delta-\varkappa)+\nu},|\xi| \geq \xi_{0},
$$

uniformly in the series $a$, and whose exact definition is of no importance. Usually, the dependence on the parameter $\rho$ is omitted from the notation.

Step I. We shall prove first that the $N$-approximation

$$
\xi^{(N)}=\xi^{(N)}(\eta ; \rho)=\eta+\sum_{j=1}^{N} \omega_{j}(\eta ; \rho)
$$

satisfies

$$
a\left(\xi^{(N)}\right)-|\eta|^{m}=u_{N+1}^{(m)},
$$

for all $\eta:|\eta| \geq \xi_{0}$. Using Taylor's formula we obtain:

$$
\begin{aligned}
\sum_{j} \mu_{j}\left(\xi^{(N)}(\eta)\right)=\sum_{j} \mu_{j}(\eta)+\sum_{j=1} \sum_{s=1}^{N} \frac{1}{s !} \partial^{s} \mu_{j}(\eta)\left(\sum_{p=1}^{N} \omega_{p}\right)^{s}+u_{N+2}^{(m)}(\eta) \\
=\sum_{j} \mu_{j}(\eta)+\sum_{s=1}^{N} \sum_{l=s}^{N} \sum_{j+p_{1}+p_{2}+\ldots p_{s}=l+1} \frac{1}{s !} \partial^{s} \mu_{j}(\eta) \omega_{p_{1}} \ldots \omega_{p_{s}}+u_{N+2}^{(m)} \\
=\sum_{j} \mu_{j}(\eta)+\sum_{l=1}^{N} \sum_{s=1}^{l} \frac{1}{s !} \sum_{j+p_{1}+p_{2}+\cdots+p_{s}=l+1} \partial^{s} \mu_{j}(\eta) \omega_{p_{1}} \ldots \omega_{p_{s}}+u_{N+2}^{(m)} \\
=\sum_{j} \mu_{j}(\eta)+\sum_{l=2}^{N} \sum_{s=1}^{l-1} \frac{1}{s !} \sum_{j+p_{1}+p_{2}+\cdots+p_{s}=l} \partial^{s} \mu_{j}(\eta) \omega_{p_{1}} \ldots \omega_{p_{s}}+u_{N+1}^{(m)} .
\end{aligned}
$$

For sufficiently large $\eta_{1}=\eta_{1}(N)$, one can assume that $\sum_{j=1}^{N}\left|\omega_{j}\right||\eta|^{-1} \leq 1 / 2$, for $|\eta| \geq \eta_{1}$ and thus the following expansion is applicable:

$$
\begin{aligned}
& \left|\xi^{(N)}\right|^{m}=|\eta|^{m}\left[1+\eta^{-1} \sum_{j=1}^{N} \omega_{j}\right]^{m} \\
& =|\eta|^{m}+m|\eta|^{m} \eta^{-1} \sum_{l=1}^{N} \omega_{l}+|\eta|^{m} \sum_{s=2}^{N} d_{s} \eta^{-s}\left(\sum_{j=1}^{N} \omega_{j}\right)^{s}+u_{N+1}^{(m)} \\
& =|\eta|^{m}+m|\eta|^{m} \eta^{-1} \sum_{l=1}^{N} \omega_{l}+\sum_{l=2}^{N} \sum_{s=2}^{l} d_{s}|\eta|^{m} \eta^{-s} \sum_{p_{1}+p_{2}+\cdots+p_{s}=l} \omega_{p_{1}} \ldots \omega_{p_{s}}+u_{N+1}^{(m)},
\end{aligned}
$$


where

$$
d_{s}=\frac{m(m-1) \cdots(m-s+1)}{s !}, s \geq 1 .
$$

Substituting these decompositions in the formula for $a\left(\xi^{(N)}\right)$, and using definitions (5.3), we arrive at (5.7).

Step II. Let us now prove that for sufficiently large $\eta$ the equation $a(\xi)=|\eta|^{m}$ has exactly one solution in a vicinity of $\eta$, and that this solution $\xi(\eta)$ satisfies $\xi(\eta)-\xi^{(N)}(\eta)=u_{N+1}^{(1)}$. Denote $T(\xi)=\sum_{j} \mu_{j}(\xi)$, and rewrite the equation in the form

$$
\xi= \pm\left(|\eta|^{m}-T(\xi)\right)^{1 / m}, \pm \eta>0 .
$$

Since $|T(\xi)| \leq C_{1}(1+|\xi|)^{\alpha+\delta}$ and $\alpha+\delta<m$, this implies that $C^{-1}\langle\eta\rangle \leq$ $\langle\xi\rangle \leq C\langle\eta\rangle$ for some $C$ uniform in $a$. Remembering also that $\left|T^{\prime}(\xi)\right| \leq$ $\tilde{C}_{1}(1+\langle\eta\rangle)^{\alpha+\delta-1}$ for such values of $\xi$, we can apply the fixed point theorem to conclude that for some $\eta_{0} \geq \xi_{0}$, depending on $C_{1}, \tilde{C}_{1}$, this equation has a unique solution $\xi(\eta)$ for each $\eta:|\eta| \geq \eta_{0}$. To prove that $\xi(\eta)$ decomposes in the asymptotic series (5.6), let us analyze the equation for the remainder $\epsilon_{N+1}(\eta)=\xi(\eta)-\xi^{(N)}(\eta)$ :

$$
\epsilon_{N+1}= \pm\left[|\eta|^{m}-T\left(\xi^{(N)}+\epsilon_{N+1}\right)\right]^{1 / m}-\xi^{(N)},
$$

assuming that $|\eta| \geq \eta_{0}$. Applying the fixed point theorem again and using the relation (5.7), we conclude that $\epsilon_{N+1}=O\left(|\eta|^{(N+1)(\delta-\varkappa)+1}\right)$, as required.

Let $a_{0}(\xi ; \rho)$ be the symbol of the operator $A_{0}$, see (5.1). Denote by $\omega_{j}(\eta ; \rho)$ the functions $\omega_{j}$ obtained from Lemma 5.1 applied to the symbol $a_{0}(\xi ; \rho)$. In the next two Lemmas we find the values of the symbols $\omega_{1}(\eta ; \rho)$ and $\omega_{2}(\eta ; \rho)$ in terms of the symbol $b(x, \xi)$. Recall the notation

$$
\mathcal{B}_{2}(\xi)=\mathcal{B}_{2}(\xi ; \rho)=\int_{0}^{2 \pi}|b(x, \xi ; \rho)|^{2} d x .
$$

We know that $\mu_{1}(\xi)=b^{o}(\xi)=(2 \pi)^{-1 / 2} \hat{b}(0, \xi)$. Now we need to find $\mu_{2}$. In the bounds below the remainders are always uniform in the symbol $b$ in the sense specified in Theorem 2.3. Note that in contrast to Section 4 we do not establish the precise dependence of the remainders on the norms $|b|_{l, s, r}^{(\alpha, \delta)}$.

Lemma 5.2 Let $b \in \mathbf{T}_{\alpha, \delta}$ with some $\alpha<m$ and $\delta<\varkappa$. Let $3 \rho / 4 \leq|\xi| \leq$ $2 \rho$. Then

$$
\begin{aligned}
\mu_{2}(\xi ; \rho)=\frac{m-1}{4 \pi m}|\xi|^{-m}\left[\mathcal{B}_{2}(\xi ; \rho)-|\hat{b}(0, \xi ; \rho)|^{2}\right] \\
\quad-\frac{1}{4 \pi m}|\xi|^{-m} \xi \partial_{\xi}\left[\mathcal{B}_{2}(\xi ; \rho)-|\hat{b}(0, \xi ; \rho)|^{2}\right]+O\left(\rho^{2(\alpha+\delta)-m-2}\right) .
\end{aligned}
$$


Proof. According to (4.11), $\mu_{2}(\xi)=\mu_{2}(\xi ; \rho)=b_{2}^{o}(\xi ; \rho)+t_{2}^{o}(\xi ; \rho)$, so that Lemma 4.3(iii) ensures that

$\mu_{2}(\xi)=-\frac{1}{2 \pi} \sum_{\theta \neq 0} \frac{|\hat{b}(\theta, \xi)|^{2}}{\tau_{\theta}(\xi)} \chi(\theta), \chi(\theta)=\chi(\theta ; \rho)=\Upsilon\left(4|\theta| \rho^{-1}\right)\left[2-\Upsilon\left(4|\theta| \rho^{-1}\right)\right]$,

for $3 \rho / 4 \leq|\xi| \leq 2 \rho$. Rewrite $\mu_{2}(\xi)=\sigma_{1}(\xi)+\sigma_{2}(\xi)$ with

$$
\begin{aligned}
& \sigma_{1}(\xi)=-\frac{1}{4 \pi} \sum_{\theta}|\hat{b}(\theta, \xi)|^{2}\left[\frac{1}{\tau_{\theta}(\xi)}+\frac{1}{\tau_{-\theta}(\xi)}\right] \chi(\theta), \\
& \sigma_{2}(\xi)=\frac{1}{4 \pi} \sum_{\theta} \frac{1}{\tau_{-\theta}(\xi)}\left[|\hat{b}(\theta, \xi)|^{2}-|\hat{b}(-\theta, \xi)|^{2}\right] \chi(\theta) .
\end{aligned}
$$

Here and below, for brevity we assume without comments that the summation runs over $\theta \neq 0$. Using the Taylor expansion for $\tau_{\theta}$ we obtain

$$
\begin{aligned}
& \frac{1}{\tau_{\theta}(\xi)}+\frac{1}{\tau_{-\theta}(\xi)}=-\frac{m-1}{m}|\xi|^{-m}+O\left(\theta^{2}|\xi|^{-m-2}\right), \\
& \frac{1}{\tau_{\theta}(\xi)}-\frac{1}{\tau_{-\theta}(\xi)}=\frac{2}{\theta m}|\xi|^{-m} \xi+O\left(\theta|\xi|^{-m-1}\right),
\end{aligned}
$$

Consequently

$$
\sigma_{1}(\xi)=\frac{m-1}{4 \pi m}|\xi|^{-m} \sum_{\theta}|\hat{b}(\theta, \xi)|^{2} \chi(\theta)+O\left(\rho^{2(\alpha+\delta)-m-2}\right) .
$$

Here we have used the inequality (2.7) with $s=0$. Using (2.7) again, we can conclude that

$$
\begin{aligned}
\sum_{\theta}|\hat{b}(\theta, \xi)|^{2} \chi(\theta)- & \sum_{\theta}|\hat{b}(\theta, \xi)|^{2} \\
& \leq \sum_{|\theta| \geq \rho / 16}|\hat{b}(\theta, \xi)|^{2}=\left(\left.|| b\right|_{l, 0} ^{(\alpha)}\right)^{2} O\left(\rho^{2(\alpha+\delta)-2 l+1}\right), \forall l>1 .
\end{aligned}
$$

By Parseval's identity

$$
\sum_{\theta \neq 0}|\hat{b}(\theta, \xi)|^{2}=\int_{0}^{2 \pi}|b(x, \xi)|^{2} d x-|\hat{b}(0, \xi)|^{2},
$$

so that

$$
\sigma_{1}(\xi)=\frac{m-1}{4 \pi m}|\xi|^{-m}\left(\mathcal{B}_{2}(\xi)-|\hat{b}(0, \xi)|^{2}\right)+O\left(\rho^{2(\alpha+\delta)-m-2}\right)+O\left(\rho^{-l}\right), \forall l>0 .
$$


To find $\sigma_{2}(\xi)$, rewrite the formula for $\sigma_{2}$ remembering that $b$ is a symmetric symbol (see $(2.5))$ and that $\chi(\theta)=\chi(-\theta)$ :

$$
\sigma_{2}(\xi)=\frac{1}{8 \pi} \sum_{\theta}\left[\frac{1}{\tau_{-\theta}(\xi)}-\frac{1}{\tau_{\theta}(\xi)}\right]\left[|\hat{b}(\theta, \xi)|^{2}-|\hat{b}(\theta, \xi-\theta)|^{2}\right] \chi(\theta) .
$$

According to the bound

$$
\left.\left|\partial_{\xi}^{s}\right| \hat{b}(\theta, \xi+\eta)\right|^{2} \mid \leq C\left(|\mathbf{|} b|_{l, s, 0}^{(\alpha, \delta)}\right)^{2} \rho^{2 \delta}\langle\xi\rangle^{2 a-s}\langle\theta\rangle^{-2 l}\langle\eta\rangle^{2|\alpha|+s},
$$

which follows from (2.7), we have

$$
\begin{aligned}
|\hat{b}(\theta, \xi)|^{2}-|\hat{b}(\theta, \xi-\theta)|^{2} & = \\
\quad & =\partial_{\xi}|\hat{b}(\theta, \xi)|^{2} \theta+\frac{1}{2} \partial_{\xi}^{2}|\hat{b}(\theta, \xi)|^{2} \theta^{2}+O\left(\rho^{2(\alpha+\delta)-3}\right)|\theta|^{-2 l+2|\alpha|+6} .
\end{aligned}
$$

Combined with (5.8), this implies that

$$
\begin{aligned}
\sigma_{2}(\xi)=-\frac{1}{4 \pi m}|\xi|^{-m} \xi \sum_{\theta} \partial_{\xi}|\hat{b}(\theta, \xi)|^{2} \chi(\theta) \\
\quad-\frac{1}{8 \pi m}|\xi|^{-m} \xi \sum_{\theta} \partial_{\xi}^{2}\left(|\hat{b}(\theta, \xi)|^{2}\right) \theta \chi(\theta)+O\left(\rho^{2(\alpha+\delta)-m-2}\right)
\end{aligned}
$$

Rewrite the second sum using (2.5):

$$
\begin{aligned}
2 \sum_{\theta} \partial_{\xi}^{2}|\hat{b}(\theta, \xi)|^{2} \theta \chi(\theta) & =\sum_{\theta} \partial_{\xi}^{2}\left(|\hat{b}(\theta, \xi)|^{2}-|\hat{b}(-\theta, \xi)|^{2}\right) \theta \chi(\theta) \\
& =\sum_{\theta} \partial_{\xi}^{2}\left(|\hat{b}(\theta, \xi)|^{2}-|\hat{b}(\theta, \xi-\theta)|^{2}\right) \theta \chi(\theta) .
\end{aligned}
$$

In view of $(2.4)$

$$
\left.\left|\partial_{\xi}^{2}\right| \hat{b}(\theta, \xi)\right|^{2}-\partial_{\xi}^{2}|\hat{b}(\theta, \xi-\theta)|^{2} \mid \leq C\left(\mid \mathbf{|} b \mathbf{|}_{l, 3,0}^{(\alpha, \delta)}\right)^{2} \rho^{2 \delta}\langle\xi\rangle^{2 \alpha-3}\langle\theta\rangle^{-2 l+2|\alpha|+4},
$$

which implies that the second term in the r.h.s. of (5.9) is of order $O\left(\rho^{2(\alpha+\delta)-m-2}\right)$. Consequently,

$$
\sigma_{2}(\xi)=-\frac{1}{4 \pi m}|\xi|^{-m} \xi \sum_{\theta} \partial_{\xi}|\hat{b}(\theta, \xi)|^{2} \chi(\theta)+O\left(\rho^{2(\alpha+\delta)-m-2}\right) .
$$

Applying Parseval's identity as in the calculation for $\sigma_{1}$ one obtains that

$$
\sigma_{2}(\xi)=-\frac{1}{4 \pi m}|\xi|^{-m} \xi \partial_{\xi}\left(\mathcal{B}_{2}(\xi)-|\hat{b}(0, \xi)|^{2}\right)+O\left(\rho^{-l}\right)+O\left(\rho^{2(\alpha+\delta)-m-2}\right),
$$

with arbitrary $l>0$. Adding up $\sigma_{1}$ and $\sigma_{2}$, one arrives at the required formula for $\mu_{2}$. 
Let us now calculate the symbols $\omega_{1}, \omega_{2}$ :

Lemma 5.3 Let $b \in \mathbf{T}_{\alpha, \delta}$ with $\alpha<m$ and $\delta<\varkappa$. For $3 \rho / 4 \leq|\eta| \leq 2 \rho$ one has

$$
\begin{aligned}
& \omega_{1}(\eta ; \rho)=-\frac{1}{m \sqrt{2 \pi}} \eta|\eta|^{-m} \hat{b}(0, \eta ; \rho), \\
& \omega_{2}(\eta ; \rho)=-\frac{m-1}{4 \pi m^{2}} \eta^{1-2 m} \mathcal{B}_{2}(\eta ; \rho)+\frac{1}{4 \pi m^{2}} \eta^{2-2 m} \partial_{\eta} \mathcal{B}_{2}(\eta ; \rho)+O\left(\rho^{2(\delta-\varkappa)-1}\right) .
\end{aligned}
$$

Proof. The symbol $\omega_{1}(\eta)$ is easy to find from (5.4) remembering that $\mu_{1}(\xi)=(2 \pi)^{-1 / 2} \hat{b}(0, \xi)$.

Let us proceed to calculating the components of the symbol (5.5). Using Lemma 5.2 , we have for $3 \rho / 4 \leq|\eta| \leq 2 \rho$

$$
\begin{aligned}
& \omega_{2}(\eta)=\omega_{2}(\eta ; \rho) \\
&=- \frac{m-1}{4 \pi m^{2}} \eta^{1-2 m}\left(\mathcal{B}_{2}(\eta)-|\hat{b}(0, \eta)|^{2}\right) \\
& \quad-\frac{m-1}{4 \pi m^{2}} \eta^{1-2 m}|\hat{b}(0, \eta)|^{2}+\frac{1}{4 \pi m^{2}} \eta^{2-2 m} \partial_{\eta}\left(\mathcal{B}_{2}(\eta)-|\hat{b}(0, \eta)|^{2}\right) \\
&+\frac{1}{4 \pi m^{2}} \eta^{2-2 m} \partial_{\eta}|\hat{b}(0, \eta)|^{2}+O\left(\rho^{2(\alpha-m+\delta)-1}\right) .
\end{aligned}
$$

This leads to the required formula.

Now we are in position to find the asymptotics of the density of states for $A_{0}$.

Theorem 5.4 (Operator $A_{0}$ ) Let $b \in \mathbf{T}_{\alpha, \delta}$ with $\alpha<m$ and $\delta<\varkappa$. Let $a_{0}(\xi ; \rho)$ be the symbol of the operator $A_{0}$ given by (5.1). Let $\eta_{0}>0$ and $\omega_{j} \in$ $\mathcal{T}_{j, \delta}^{1}\left(\xi_{0}, 1\right), \forall \xi_{0}>0$, be the number and symbols from Lemma 5.1 constructed for the symbol $a_{0}(\xi ; \rho)$. Let

$$
\xi_{M}(\eta ; \rho)=\eta+\sum_{j=1}^{M} \omega_{j}(\eta ; \rho) .
$$

Then the integrated density of states $D\left(\eta^{m} ; A_{0}\right)$ and the Fourier coefficients $\hat{N}\left(\eta^{m}, 2 \pi n ; A_{0}\right), \eta \geq 0$, satisfy the formulas

$$
\begin{gathered}
(5.11) \quad D\left(\eta^{m} ; A_{0}\right)=\frac{1}{2 \pi}\left[\xi_{M}(\eta ; \rho)-\xi_{M}(-\eta ; \rho)\right]+O\left(\langle\rho\rangle^{(M+1)(\delta-\varkappa)+1}\right) \\
\hat{N}\left(\eta^{m}, 2 \pi n ; A_{0}\right)=-\frac{1}{4 \pi^{2} i n}\left[\exp \left(-2 \pi i n \xi_{M}(\eta ; \rho)\right)-\exp \left(-2 \pi i n \xi_{M}(-\eta ; \rho)\right)\right] \\
+O\left(\langle\rho\rangle^{(M+1)(\delta-\varkappa)+1}\right) .
\end{gathered}
$$

for all $3 \rho / 4 \leq \eta \leq 2 \rho$ and $\rho \geq 1+4 \eta_{0} / 3$. The functions $\omega_{1}$, $\omega_{2}$ satisfy the formulas (5.4), (5.5). 
Proof. Let $\xi( \pm \eta ; \rho)$ be the function constructed in Lemma 5.1 for $\eta \geq \eta_{0}$. According to Proposition 2.1

$$
\begin{gathered}
2 \pi D\left(\eta^{m} ; A_{0}\right)=\xi(\eta ; \rho)-\xi(-\eta ; \rho), \\
\hat{N}\left(\eta^{m}, 2 \pi n ; A_{0}\right)=-\frac{1}{4 \pi^{2} i n}(\exp (-2 \pi i n \xi(\eta ; \rho))-\exp (-2 \pi i n \xi(-\eta ; \rho))), n \neq 0,
\end{gathered}
$$

for all $\eta \geq \eta_{0}$. In particular, the function $\xi( \pm \eta ; \rho)$ is well defined for $\eta \in$ $[3 \rho / 4,2 \rho]$ since $\rho \geq 1+4 \eta_{0} / 3$. In view of Lemma 5.1 for $3 \rho / 4 \leq \eta \leq 2 \rho$ we have $\xi( \pm \eta ; \rho)=\xi_{M}( \pm \eta ; \rho)+O\left(\rho^{(M+1)(\delta-\varkappa)+1}\right)$, which leads to (5.11) and (5.12). Besides, the first two components $\omega_{1}, \omega_{2}$ are given by the required formulas due to Lemma 5.3.

\subsection{Operator $A_{1}$}

Now it is time to understand how the density of states behaves for the perturbed operator, i.e. $A_{1}$. The first step is to establish some bounds relating the counting functions of the fibre operators $A_{0}(k)$ and $A_{1}(k)$ for $k \in(0,1)$. Recall that $N\left(\rho^{m}, k ; A_{0}\right)$ and $N\left(\rho^{m}, k ; A_{1}\right)$ are finite since the operators $A_{0}(k)$, and $A_{1}(k)$ have purely discrete spectra (see Section 2).

Lemma 5.5 Let $b \in \mathbf{T}_{\alpha, \delta}$ with $\alpha_{1}+\delta<m$ where $\alpha_{1}=\max (0, \alpha)$. Then for any $M>0$ there exists a number $\rho_{0} \geq 1$ bounded from above uniformly in $b$, such that

$$
\begin{gathered}
N\left(\lambda_{-}, k ; A_{0}\right) \leq N\left(\rho^{m}, k ; A_{1}\right) \leq N\left(\lambda_{+}, k ; A_{0}\right), \\
\lambda_{ \pm}=\rho^{m} \pm c \rho^{(M+1)(\delta-\varkappa)+m}, \quad \varkappa=m-\alpha,
\end{gathered}
$$

for all $\rho \geq \rho_{0}$ and all $k \in[0,1]$. The constant $c$ is uniform in $b$ and depends on the parameter $M$.

Proof. In view of the bound $\left\|R_{M+1}(H)\right\| \leq C \rho^{(M+1)(\delta-\varkappa)+m}$ established in Lemma 4.3, we have

$$
N\left(\lambda_{-}, k ; A_{0}+L^{b, \sharp, \uparrow}\right) \leq N\left(\rho^{m}, k ; A_{1}\right) \leq N\left(\lambda_{+}, k ; A_{0}+L^{b, \sharp, \uparrow}\right), L=L_{M}
$$

To get rid of $L^{b, \sharp, \uparrow}$ in (5.13), represent $A_{0}+L^{b, \sharp, \uparrow}$ in a matrix form by decoupling the space $\mathcal{H}=\mathrm{L}^{2}(\mathbb{R})$ in the orthogonal sum of three subspaces corresponding to the following three disjoint subsets of $\mathbb{R}$ : $\Lambda_{r}, \Omega_{R}$ (see (3.16) for definition) and $\Upsilon_{r, R}=\Omega_{r} \cap \Lambda_{R}$, where $0<r<R<\infty$ with $r=11 \rho / 16$ and $R=2 \rho$. Denote

$$
\mathcal{P}_{1}=\mathcal{P}\left(\Lambda_{r}\right), \mathcal{P}_{2}=\mathcal{P}\left(\Upsilon_{r, R}\right), \mathcal{P}_{3}=\mathcal{P}\left(\Omega_{R}\right),
$$


so that $I=\mathcal{P}_{1}+\mathcal{P}_{2}+\mathcal{P}_{3}$. By Lemma 3.5 we have

$$
\left(\mathcal{P}_{2}+\mathcal{P}_{3}\right) L^{b}=L^{b}\left(\mathcal{P}_{2}+\mathcal{P}_{3}\right)=\left(\mathcal{P}_{1}+\mathcal{P}_{2}\right) L^{\sharp}=L^{\sharp}\left(\mathcal{P}_{1}+\mathcal{P}_{2}\right)=0 .
$$

Therefore $A_{0}+L^{b, \sharp, \uparrow}$ takes the form $\tilde{A}_{0}+\tilde{L}$ with

$$
\begin{gathered}
\tilde{A}_{0}=\left[\begin{array}{ccc}
\mathcal{P}_{1}\left(A_{0}+L^{b}\right) \mathcal{P}_{1} & 0 & 0 \\
0 & A_{0} \mathcal{P}_{2} & 0 \\
0 & 0 & \mathcal{P}_{3}\left(A_{0}+L^{\sharp, \uparrow}\right) \mathcal{P}_{3}
\end{array}\right], \\
\tilde{L}=\left[\begin{array}{ccc}
\mathcal{P}_{1} L^{\uparrow} \mathcal{P}_{1} & \mathcal{P}_{1} L^{\uparrow} \mathcal{P}_{2} & \mathcal{P}_{1} L^{\uparrow} \mathcal{P}_{3} \\
\mathcal{P}_{2} L^{\uparrow} \mathcal{P}_{1} & \mathcal{P}_{2} L^{\uparrow} \mathcal{P}_{2} & \mathcal{P}_{2} L^{\uparrow} \mathcal{P}_{3} \\
\mathcal{P}_{3} L^{\uparrow} \mathcal{P}_{1} & \mathcal{P}_{3} L^{\uparrow} \mathcal{P}_{2} & 0
\end{array}\right]
\end{gathered}
$$

By (4.10), Lemma 4.3 and Lemma 3.5(iv), the norm of the operator $\tilde{L}$ is bounded by $C_{l} \rho^{-l}$ with arbitrary $l>0$ uniformly in $b$. Therefore the estimate (5.13) transforms into the two-sided bound

$$
N\left(\lambda_{-}, k ; \tilde{A}_{0}\right) \leq N\left(\rho^{m}, k ; A_{1}\right) \leq N\left(\lambda_{+}, k ; \tilde{A}_{0}\right) .
$$

Notice that $\lambda_{+} \leq(5 \rho / 4)^{m}, \lambda_{-} \geq(4 \rho / 5)^{m}$ for a sufficiently large $\rho_{0}$. Thus the proof of (5.14) will be completed if we show that

$$
\begin{gathered}
N\left(\mu^{m}, k ; \mathcal{P}_{1}\left(A_{0}+L^{b}\right) \mathcal{P}_{1}\right)=N\left(\mu^{m}, k ; \mathcal{P}_{1} A_{0}\right), \\
N\left(\mu^{m}, k ; \mathcal{P}_{3}\left(A_{0}+L^{\sharp, \uparrow}\right) \mathcal{P}_{3}\right)=N\left(\mu^{m}, k ; \mathcal{P}_{3} A_{0} \mathcal{P}_{3}\right)=0,
\end{gathered}
$$

for all $\mu \in[4 \rho / 5,5 \rho / 4]$. According to (4.17),

$$
\sum_{l=1}^{M}\left|b_{l} \mathbf{|}^{(\alpha, \delta)}+\sum_{l=2}^{M}\right| t_{l} \mathbf{|}^{(\alpha, \delta)} \leq C .
$$

Thus by virtue of (3.15) and (3.3) we obtain

$$
\left|\left(\left(L^{\sharp}+L^{\uparrow}\right) u, u\right)\right| \leq C \rho^{\delta}\left(\Xi^{\alpha} u, u\right), \Xi=\mathrm{Op}(\langle\xi\rangle),
$$

and hence

$$
\left(\left(A_{0}+L^{\sharp}+L^{\uparrow}\right) \mathcal{P}_{3} u, \mathcal{P}_{3} u\right) \geq\left(2^{m}\left(\rho^{m}-C \rho^{\alpha+\delta}\right) \mathcal{P}_{3} u, \mathcal{P}_{3} u\right) \geq(3 \rho / 2)^{m}\left\|\mathcal{P}_{3} u\right\|^{2} .
$$

Here we have used the fact that $\alpha+\delta<m$. Therefore the fibre $\left(\mathcal{P}_{3}\left(A_{0}+\right.\right.$ $\left.\left.L^{\sharp}+L^{\uparrow}\right) \mathcal{P}_{3}\right)(k)$ satisfies the same bound for a.a. $k$. This ensures (5.16). Similarly, by Lemma 3.5(i)

$$
\left\|L^{b}\right\| \leq C \rho^{\alpha_{1}+\delta}, \alpha_{1}=\max (\alpha, 0) .
$$

The operator $A_{0} \mathcal{P}_{1}$ satisfies $A_{0} \mathcal{P}_{1} \leq(3 \rho / 4)^{m}$ for $\rho \geq \rho_{0}$. Since $\alpha_{1}+\delta<m$, the norm $\left\|L^{b}\right\|$ is strictly less than $(4 \rho / 5)^{m}-(3 \rho / 4)^{m}$. Now a straightforward perturbation argument leads to (5.15). This completes the proof of (5.14) and that of the Lemma.

Thus the question is reduced to the study of the operator $A_{0}$. 


\subsection{Proof of Theorem 2.4}

Increasing if necessary the value of $\rho_{0}=\rho_{0}(M)$ from Lemma 5.5, we assume that $\rho_{0} \geq 1+4 \eta_{0} / 3$, where $\eta_{0}$ is the constant from Theorem 5.4. Consequently, in view of Lemma 5.5 and Theorem 5.4, for $\rho \geq \rho_{0}$ and any $n \in \mathbb{Z}$ we have

$$
\begin{aligned}
\mid \hat{N}\left(\rho^{m}, 2 \pi n ; A_{1}\right) & -\hat{N}\left(\lambda_{-}, 2 \pi n ; A_{0}\right)\left|\leq \frac{1}{2 \pi} \int_{0}^{1}\right| N\left(\rho^{m}, k ; A_{1}\right)-N\left(\lambda_{-}, k ; A_{0}\right) \mid d k \\
= & D\left(\rho^{m}, A_{1}\right)-D\left(\lambda_{-}, A_{0}\right) \\
\leq & D\left(\lambda_{+}, A_{0}\right)-D\left(\lambda_{-}, A_{0}\right)=O\left(\rho^{(\delta-\varkappa)(M+1)+1}\right) .
\end{aligned}
$$

For the last estimate we have used the fact that $\omega_{j} \in \mathcal{T}_{j, \delta}^{1}\left(\xi_{0}, 1\right)$ in the formula (5.10). For the same reason, by Theorem 5.4 we have

$$
\begin{aligned}
\hat{N}\left(\rho^{m}, 2 \pi n ; A_{1}\right) & =\hat{N}\left(\lambda_{-}, 2 \pi n ; A_{0}\right)+O\left(\rho^{(\delta-\varkappa)(M+1)+1}\right) \\
& =\hat{N}\left(\rho^{m}, 2 \pi n ; A_{0}\right)+O\left(\rho^{(\delta-\varkappa)(M+1)+1}\right), \quad \forall n \in \mathbb{Z} .
\end{aligned}
$$

Since the unitary operator $U=\exp (i \Psi)$ is $\Gamma$-periodic, by Lemma 2.2

$$
\hat{N}\left(\rho^{m}, 2 \pi n ; A_{1}\right)=\hat{N}\left(\rho^{m}, 2 \pi n ; H\right) .
$$

To find $\hat{N}\left(\rho^{m}, 2 \pi n ; A_{0}\right)$ use Theorem 5.4 again. This leads to the formulas $(2.14)$ and $(2.15)$ for $\rho \geq \rho_{0}$, if one denotes $\nu_{j}(\xi):=(1-\Upsilon(\rho / 2)) \omega_{j}(\xi ; \rho)$, $\xi= \pm \rho$, see (3.6) for definition of the function $\Upsilon$. In view of Theorem 5.4, $\nu_{1}, \nu_{2}$ satisfy the required formulas and $\nu_{j} \in \mathcal{S}_{j, \delta}^{1}$.

To obtain formulas $(2.14),(2.15)$ for $\rho \leq \rho_{0}$ we need to check that

$$
\left|\hat{N}\left(\rho^{m}, 2 \pi n\right)\right| \leq C, n \in \mathbb{Z},
$$

with a constant $C$ uniform in $b$. This is done in Remark 2.5.

As was explained previously, Theorem 2.4 leads to Theorem 2.3.

\section{Acknowledgments}

The first draft of the paper was completed during the author's stay at the Mittag-Leffler Institute in September 2002. The author thanks

- G. Rosenblum for information concerning A. Milevskiı's work and for kindly supplying manuscripts of A. Milevskiì's papers,

- the referee for useful remarks and for drawing his attention to paper [16],

- and his PhD student D. Hughes who has carefully read the manuscript and whose remarks helped to improve the text. 


\section{References}

[1] Agranovich, M. S.: Elliptic operators on closed manifolds. (Russian) In Current problems in mathematics. Fundamental directions 63, 5-129. Itogi Nauki i Tekhniki, Akad. Nauk SSSR, Vsesoyuz. Inst. Nauchn. i Tekhn. Inform., Moscow, 1990. English translation in: Partial differential equations VI. Elliptic and Parabolic Operators, 1-130. Encycl. Math. Sci. 63, Springer, 1994.

[2] Dahlberg, B. E. J. and Trubowitz, E.: A remark on two dimensional periodic potentials. Comment. Math. Helv. 57 (1982), 130-134.

[3] Feldman, J., Knörrer, H. and Trubowitz, E.: Perturbatively unstable eigenvalues of a periodic Schrödinger operator. Comment. Math. Helv. 66 (1991), 557-579.

[4] Helffer, B. And Mohamed, A.: Asymptotic of the density of states for the Schrödinger operator with periodic electric potential. Duke Math. J. 92 (1998), 1-60.

[5] Karpeshina, Yu.: On the density of states for the periodic Schrödinger operator. Ark. Mat. 38 (2000), 111-137.

[6] Karpeshina, Yu.: Perturbation theory for the Schrödinger operator with a periodic potential. Lecture Notes in Mathematics 1663. Springer-Verlag, Berlin, 1997.

[7] Milevskiř, A. S.: Similarity transformations and spectral properties of hypoelliptic pseudodifferential operators on the circle. (Russian) Funktsional. Anal. i Prilozhen. 23 (1989), no. 3, 71-72. English translation in Funct. Anal. Appl. 23 (1989), no. 3, 231-233.

[8] Milevskil, A.S.: A simplification of hypoelliptic psedodifferential operators on the circle via Fourier integral operators. Dep. in VINITI 05.09.88, No 6856-B88, Moscow, 1988.

[9] Parnovski, L. And Sobolev, A.V.: Lattice points, perturbation theory and the periodic polyharmonic operator. Ann. Henri Poincaré 2 (2001), $573-581$.

[10] Reed, M. And Simon, B.: Methods of modern mathematical physics, IV. Analysis of operators. Academic Press, New York-London, 1975.

[11] Rozenbluum, G.V.: Near-similarity of operators and the spectral asymptotic behavior of pseudodifferential operators on the circle. (Russian) Trudy Moskov. Mat. Obshch. 36 (1978), 59-84.

[12] Schenk, D. And Shubin, M. A.: Asymptotic expansion of state density and the spectral function of the Hill operator. Math. USSR-Sb. 56 (1987), no. $2,492-515$.

[13] Shubin, M. A.: Weyl's theorem for the Schrödinger operator with an almost periodic potential. Vestnik Moskov. Univ. Ser. I Mat. Mekh. 31 (1976), no. 2, 84-88 (Russian). English translation in Moscow Univ. Math. Bull. 31 (1976), 133-137. 
[14] Shubin, M. A.: The spectral theory and the index of elliptic operators with almost periodic coefficients. Russian Math Surveys 34 (1979), no 2, 109-157.

[15] Skriganov, M.: Geometric and arithmetic methods in the spectral theory of the multi-dimensional periodic operators. (Russian) Trudy Mat. Inst. Steklov. 171 (1985), 122 pp.

[16] Weinstein, A.: Asymptotics of eigenvalue clusters for the Laplacian plus a potential. Duke Math. J. 44 (1977), 883-892.

Recibido: 21 de enero de 2003

Revisado: 8 de marzo de 2004

Alexander V. Sobolev

School of Mathematical Sciences

Watson Building University of Birmingham

Edgbaston, Birmingham

B15 2TT, UK

a.sobolev@bham.ac.uk 